\title{
Discrete flavor symmetries and models of neutrino mixing
}

\author{
Guido Altarelli* \\ Dipartimento di Fisica "E. Amaldi," Università di Roma Tre INFN, Sezione di Roma Tre, \\ I-00146 Rome, Italy \\ and Department of Physics, Theory Division, CERN, CH-1211 Geneva 23, Switzerland
}

\author{
Ferruccio Feruglio ${ }^{\dagger}$ \\ Dipartimento di Fisica "G. Galilei," Università di Padova INFN, Sezione di Padova, \\ Via Marzolo 8, I-35131 Padua, Italy
}

(Published 16 September 2010)

\begin{abstract}
Application of non-Abelian finite groups to the theory of neutrino masses and mixing is reviewed, which is strongly suggested by the agreement of the tribimaximal (TB) mixing pattern with experiment. After summarizing the motivation and the formalism, concrete models based on $A_{4}, S_{4}$, and other finite groups, and their phenomenological implications are discussed, including lepton flavor violating processes, leptogenesis, and the extension to quarks. As an alternative to TB mixing application of discrete flavor symmetries to quark-lepton complementarity and bimaximal mixing is also considered.
\end{abstract}

DOI: 10.1103/RevModPhys.82.2701

PACS number(s): 14.60.Pq, 11.30.-j

\section{CONTENTS}

\author{
I. Introduction \\ II. Special Patterns of Neutrino Mixing \\ III. The $A_{4}$ Group \\ IV. Applying $A_{4}$ to Lepton Masses and Mixings \\ V. Possible Origin of $A_{4}$ \\ VI. Alternative Routes to TB Mixing \\ VII. Extension to Quarks and GUTs \\ A. Extension to quarks without GUTs \\ B. Extension to quarks within GUTs \\ VIII. The $S_{4}$ Group and BM Mixing \\ IX. Lepton Flavor Violation \\ $\mathrm{X}$. Leptogenesis \\ XI. Summary and Conclusion \\ Acknowledgments \\ References
}

\section{INTRODUCTION}

Experiments on neutrino oscillations, which measure differences between squared masses and mixing angles (Altarelli and Feruglio, 2004; Grimus, 2006; Mohapatra and Smirnov, 2006; Mohapatra et al., 2007; GonzalezGarcia and Maltoni, 2008a; Altarelli, 2009), have established that neutrinos have a mass. We refer the interested reader, in particular, to Altarelli and Feruglio (2004) for an introduction to the subject, the main results, the basic formalism, and all definitions and notations. Two distinct oscillation frequencies have first been measured in solar (Hosaka et al., 2006a; Cravens et al.,

\footnotetext{
*guido.altarelli@cern.ch

†feruglio@pd.infn.it
}

2008; Aharmim et al., 2010) and atmospheric (Abe et al., 2006; Hosaka et al., 2006b) neutrino oscillations and later confirmed by experiments on Earth, such as KamLAND (Abe et al., 2008), K2K (Ahn et al., 2006), MINOS (Kafka, 2010), and OPERA (Agafonova et al., 2010). A signal corresponding to a third mass difference was claimed by the LSND experiment (Athanassopoulos et al., 1996, 1998a, 1998b) but not confirmed by KARMEN (Armbruster et al., 2002) and recently by MiniBooNE (Aguilar-Arevalo et al., 2009a, 2009b). Two well separated differences need at least three different neutrino mass eigenstates involved in oscillations. Actually, the three known neutrino species can be sufficient. At least two $\nu$ 's must be massive while, in principle, the third one could still be massless. In the following, we assume the simplest picture with three active neutrinos, no sterile neutrinos, and CPT invariance. The mass eigenstates involved in solar oscillations are $m_{1}$ and $m_{2}$ and, by definition, $\left|m_{2}\right|>\left|m_{1}\right|$, so that $\Delta m_{\text {sun }}^{2}=\Delta m_{21}^{2}$ $=\left|m_{2}\right|^{2}-\left|m_{1}\right|^{2}>0$. The atmospheric neutrino oscillations involve $m_{3}: \Delta m_{\mathrm{atm}}^{2}=\left|\Delta m_{31}^{2}\right|$ with $\Delta m_{31}^{2}=\left|m_{3}\right|^{2}-\left|m_{1}\right|^{2}$ either positive (normal hierarchy) or negative (inverse hierarchy). The present data (Strumia and Vissani, 2006; Bandyopadhyay et al., 2008; Fogli et al., 2008a, 2008b; Gonzalez-Garcia and Maltoni, 2008b; Maltoni and Schwetz, 2008; Schwetz et al., 2008) are compatible with both cases. The degenerate spectrum occurs when the average absolute value of the masses is much larger than all mass squared differences: $\left|m_{i}\right|^{2} \gg\left|\Delta m_{h k}^{2}\right|$. With the standard set of notations and definitions (Altarelli and Feruglio, 2004) the present data are summarized in Table I.

Oscillation experiments do not provide information about either the absolute neutrino mass scale or the Dirac or Majorana nature of neutrinos. Limits on the mass scale are obtained (Altarelli and Feruglio, 2004) 
TABLE I. Fits to neutrino oscillation data.

\begin{tabular}{lcc}
\hline \hline Quantity & $\begin{array}{c}\text { Fogli } \text { et al., 2008a, } \\
2008 \mathrm{~b}\end{array}$ & $\begin{array}{c}\text { Maltoni and Schwetz, 2008; } \\
\text { Schwetz } \text { et al., 2008 }\end{array}$ \\
\hline$\Delta m_{\text {sun }}^{2}\left(10^{-5} \mathrm{eV}^{2}\right)$ & $7.67_{-0.19}^{+0.16}$ & $7.65_{-0.20}^{+0.23}$ \\
$\Delta m_{\text {atm }}^{2}\left(10^{-3} \mathrm{eV}^{2}\right)$ & $2.39_{-0.08}^{+0.11}$ & $2.40_{-0.11}^{+0.12}$ \\
$\sin ^{2} \theta_{12}$ & $0.312_{-0.018}^{+0.019}$ & $0.304_{-0.016}^{+0.022}$ \\
$\sin ^{2} \theta_{23}$ & $0.466_{-0.058}^{+0.073}$ & $0.50_{-0.06}^{+0.07}$ \\
$\sin ^{2} \theta_{13}$ & $0.016 \pm 0.010$ & $0.010_{-0.011}^{+0.016}$ \\
\hline \hline
\end{tabular}

from the end point of the tritium beta decay spectrum, from cosmology [see, for example, Lesgourgues and Pastor (2006)], and from neutrinoless double beta decay $(0 \nu \beta \beta)$ [for a recent review, see, for example, Avignone et al. (2008)]. From tritium we have an absolute upper limit of $2.2 \mathrm{eV}$ [at $95 \%$ confidence limit (C.L.)] on the mass of electron antineutrino (Kraus et al., 2005), which, combined with the observed oscillation frequencies under the assumption of three $C P T$-invariant light neutrinos, also represents an upper bound on the masses of the other active neutrinos. Complementary information on the sum of neutrino masses is also provided by the galaxy power spectrum combined with measurements of the cosmic microwave background anisotropies. According to recent analyses of the most reliable data (Fogli et al., 2008) $\Sigma_{i}\left|m_{i}\right|<0.60-0.75 \mathrm{eV}$ (at $95 \%$ C.L.) depending on the retained data (the numbers for the sum have to be divided by 3 in order to obtain a limit on the mass of each neutrino). The discovery of $0 \nu \beta \beta$ decay would be important because it would establish lepton number violation and the Majorana nature of $\nu$ 's as well as provide direct information on the absolute scale of neutrino masses. The present limit from $0 \nu \beta \beta$ (with large ambiguities from nuclear matrix elements) is about $\left|m_{e e}\right|$ $<0.3-0.8 \mathrm{eV}$ (Avignone et al., 2008; Fogli et al., 2008) [see Eq. (4)].

After KamLAND (Abe et al., 2008), SNO (Aharmim et al., 2010), and the upper limits on the absolute value of neutrino masses, not too much hierarchy in the spectrum of neutrinos is indicated by experiments,

$$
r=\Delta m_{\mathrm{sol}}^{2} / \Delta m_{\mathrm{atm}}^{2} \sim 1 / 30 .
$$

Precisely $r=0.032_{-0.005}^{+0.006}$ at $3 \sigma$ 's (Fogli et al., 2008a, 2008b; Maltoni and Schwetz, 2008; Schwetz et al., 2008). Thus, for a hierarchical spectrum, $m_{2} / m_{3} \sim \sqrt{r} \sim 0.2$, which is comparable to the Cabibbo angle $\lambda_{C} \sim 0.22$ or to its leptonic analog $\sqrt{m_{\mu} / m_{\tau}} \sim 0.24$. This suggests that the same hierarchy parameter [raised to powers with $\mathcal{O}(1)$ exponents] may apply for quark, charged-lepton, and neutrino mass matrices. This, in turn, indicates that, in the absence of some special dynamical reason, we do not expect quantities such as $\theta_{13}$ or the deviation of $\theta_{23}$ from its maximal value to be too small. Indeed, it would be important to know how small the mixing angle $\theta_{13}$ is and how close to maximal $\theta_{23}$ is.

Given that neutrino masses are certainly extremely small, it is really difficult from the theory point of view to avoid the conclusion that the lepton number $L$ conservation is probably violated and that $\nu$ 's are Majorana fermions. In this case, the small size of neutrino masses can be naturally explained as inversely proportional to the large scale where $L$ conservation is violated. If neutrinos are Majorana particles, their masses arise from the generic dimension-five nonrenormalizable operator of the form (Weinberg, 1979)

$$
O_{5}=\frac{(H l)_{i}^{T} \eta_{i j}(H l)_{j}}{M}+\text { H.c. },
$$

with $H$ being the ordinary Higgs doublet, $l_{i}$ being the $\mathrm{SU}(2)$ lepton doublets, $\eta$ being a matrix in flavor space, and $M$ being a large scale of mass, and a charge conjugation matrix $C$ between the lepton fields is understood. For $\eta_{i j} \approx \mathcal{O}(1)$, neutrino masses generated by $O_{5}$ are of the order $m_{\nu} \approx v^{2} / M$ where $v \sim O(100 \mathrm{GeV})$ is the vacuum expectation value of the ordinary Higgs. A particular realization of this effective mass operator is given by the seesaw mechanism (Minkowski, 1977; Gell-Mann et al., 1979; Yanagida, 1979; Glashow, 1980; Mohapatra and Senjanovic, 1980), where $M$ is derived from the exchange of heavy neutral objects of weak isospin 0 or 1 . In the simplest case the exchanged particle is the righthanded $(\mathrm{RH})$ neutrino $\nu^{c}$ (a gauge singlet fermion described here through its charge conjugate field), and the resulting neutrino mass matrix reads (type I seesaw) (Altarelli and Feruglio, 2004)

$$
m_{\nu}=m_{D}^{T} M^{-1} m_{D},
$$

where $m_{D}$ and $M$ denote the Dirac neutrino mass matrix (defined as $\nu^{c T} m_{D} \nu$ ) and the Majorana mass matrix of $\nu^{c}$ (defined as $\nu^{c T} M \nu^{c}$ ), respectively. As one sees, the light neutrino masses are quadratic in the Dirac masses and inversely proportional to the large Majorana mass. For $m_{\nu} \approx \sqrt{\Delta m_{\mathrm{atm}}^{2}} \approx 0.05 \mathrm{eV}$ and $m_{\nu} \approx m_{D}^{2} / M$ with $m_{D} \approx v$ $\approx 200 \mathrm{GeV}$, we find $M \approx 10^{15} \mathrm{GeV}$ which, indeed, is an impressive indication that the scale for lepton number violation is close to the grand unified scale $M_{\mathrm{GUT}}$. Thus, neutrino masses are probably a probe into the physics near $M_{\mathrm{GUT}}$. This argument, in our opinion, strongly discourages models where neutrino masses are generated near the weak scale and are suppressed by some special mechanism.

Oscillation experiments cannot distinguish between Dirac and Majorana neutrinos. The detection of neu- 
Full dependence on $\min m_{\nu}$

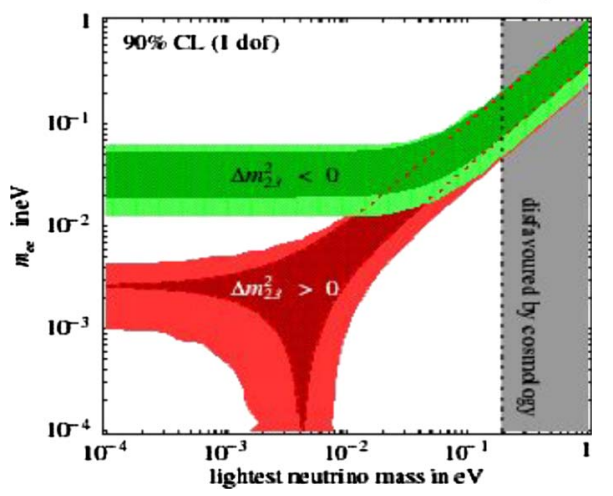

FIG. 1. (Color online) A plot of $m_{e e}$ in eV, the quantity measured in neutrinoless double beta decay, given in Eq. (4), vs the lightest neutrino mass $m_{1}$, also in $\mathrm{eV}$. The upper (lower) band is for inverse (normal) hierarchy. From Feruglio et al., 2002.

trinoless double beta decay would provide direct evidence of $L$ nonconservation and the Majorana nature of neutrinos. It would also offer a way to possibly disentangle the three cases of degenerate, normal, or inverse hierarchy neutrino spectrum. The quantity, which is bound by experiments on $0 \nu \beta \beta$, is the 11 entry of the $\nu$ mass matrix, which in general, from $m_{\nu}=U^{*} m_{\text {diag }} U^{\dagger}$, is given by

$$
\left|m_{e e}\right|=\left|\left(1-s_{13}^{2}\right)\left(m_{1} c_{12}^{2}+m_{2} s_{12}^{2}\right)+m_{3} e^{2 i \phi} s_{13}^{2}\right|,
$$

where $U \equiv U_{\text {PMNS }}$ is the mixing matrix, $m_{1,2}$ are complex masses (including the Majorana phases) while $m_{3}$ can be taken as real and positive, and $\phi$ is the $U_{\text {PMNS }}$ phase measurable from $C P$ violation in oscillation experiments. Starting from this general formula it is simple to derive the bounds for degenerate, inverse hierarchy, or normal hierarchy mass patterns shown in Fig. 1 (Feruglio et al., 2002). In the next few years a new generation of experiments will reach a larger sensitivity on $0 \nu \beta \beta$ by about an order of magnitude. If these experiments observe a signal, this will be compatible with both types of neutrino mass ordering; if not, then the normal hierarchy case remains a possibility. Establishing that $L$ is violated in particle interactions would also strongly support the possibility that the observed baryon asymmetry is generated via leptogenesis through the out of equilibrium, $C P$, and $L$ violating decays of the heavy $\mathrm{RH}$ neutrinos (see Sec. X).

Neutrino mixing is important because it could, in principle, provide new clues for the understanding of the flavor problem. Even more so since neutrino mixing angles show a pattern that is completely different from that of quark mixing: for quarks all mixing angles are small, for neutrinos two angles are large (one is even compatible with the maximal value), and only the third one is small. For building up theoretical models of neutrino mixing one must guess which features of the data are really relevant in order to identify the basic principles for the formulation of the model. In particular, it is an experimental fact (Strumia and Vissani, 2006; Ban- dyopadhyay et al., 2008; Fogli et al., 2008a, 2008b; Gonzalez-Garcia and Maltoni, 2008b; Maltoni and Schwetz, 2008; Schwetz et al., 2008) that within measurement errors the observed neutrino mixing matrix (Altarelli and Feruglio, 2004) is compatible with the socalled tribimaximal (TB) form in Eq. (12) (Harrison and Scott, 2002, 2003, 2004; Harrison et al., 2002). The best measured neutrino mixing angle $\theta_{12}$ is just about $1 \sigma$ below the TB value $\tan ^{2} \theta_{12}=1 / 2$, while the other two angles are well inside the $1 \sigma$ interval (see Table I). Thus, one possibility is that one takes this coincidence seriously and only considers models where TB mixing is automatically a good first approximation. Alternatively, one can assume that the agreement of the data with TB mixing is accidental. Indeed, there are many models that fit the data and yet TB mixing does not play any role in their architecture. For example, from Albright and Rodejohann (2008), there is a list of grand unified $\mathrm{SO}(10)$ models with parameters that can be fitted to the neutrino mixing angles, leading to a good agreement with the data, although most of these models have no built-in relation with TB mixing [see also Bertolini et al. (2006)]. Another class of examples is from Plentinger and Seidl (2008). Clearly, for this type of models, in most cases, different mixing angles could also be accommodated by simply varying the fitted values of the parameters. If instead we assume that TB mixing has a real physical meaning, then we are led to consider models that naturally produce TB mixing in first approximation and only a special dynamics can lead to this peculiar mixing matrix. Discrete non-Abelian groups [for an introduction see, for example, Frampton and Kephart (1995) and Ishimori et al. (2010)] naturally emerge as suitable flavor symmetries. In fact, the TB mixing matrix immediately suggests rotations by fixed discrete angles. It has been found that a broken flavor symmetry based on the discrete group $A_{4}$ (the group of even permutations of 4 elements, which can be seen as the invariance group of a rigid regular tetrahedron) appears to be particularly suitable to reproduce this specific mixing pattern in leading order (LO). A nonexhaustive list of papers that discuss the application of $A_{4}$ to neutrino mixing is given by Ma and Rajasekaran (2001) Babu et al. (2003), Hirsch et al. (2004, 2005, 2007, 2008), Ma (2004a, 2004b, 2005a, 2005b, 2005c, 2006b, 2006c, 2007), Altarelli and Feruglio (2005, 2006), Babu and $\mathrm{He}$ (2005), Chen et al. (2005), Zee (2005), Adhikary et al. (2006), He et al. (2006), Ma et al. (2006), Altarelli et al. (2007, 2008), Lavoura and Kuhbock (2007), Morisi et al. (2007), Yin (2007), Adhikary and Ghosal (2008), Bazzocchi, Frigerio, and Morisi (2008), Bazzocchi, Kaneko, and Morisi (2008), Bazzocchi, Morisi, and Picariello (2008), Brahmachari et al. (2008), Csaki et al. (2008), Frampton and Matsuzaki (2008), Grimus and Kuhbock (2008), Honda and Tanimoto (2008), Altarelli and Meloni (2009), Bazzocchi, Morisi, Picariello, and Torrente-Lujan (2009), Ciafaloni et al. (2009), Lin (2009a, 2009b), Morisi (2009), Antusch et al. (2010), del Aguila et al. (2010), and Kadosh and Pallante (2010). The choice of this particular discrete group is not unique and, for example, other so- 
lutions based on alternative discrete flavor symmetries [see, for example, $T^{\prime}$ (Frampton and Kephart, 1995, 2007; Aranda et al., 2000a, 2000b; Aranda, 2007; Carr and Frampton, 2007; Chen and Mahanthappa, 2007; Feruglio et al., 2007; Ding, 2008; Frampton and Matsuzaki, 2009), $S_{4}$ (Mohapatra et al., 2004; Cai and Yu, 2006; Hagedorn et al., 2006, 2010; Ma, 2006a; Bazzocchi and Morisi, 2009; Bazzocchi et al., 2009a, 2009b; Dutta et al., 2009, 2010; Ishimori et al., 2009, 2010; Ding, 2010; Meloni, 2010; Morisi and Peinado, 2010), $\Delta$ (27) (de Medeiros Varzielas et al., 2007a; Luhn et al., 2007b; Grimus and Lavoura, 2008; Ma, 2008; Bazzocchi and de Medeiros Varzielas, 2009; Ding, 2010), and other groups (Luhn et al., 2007a, 2007c; Everett and Stuart, 2009; King and Luhn, 2009a, 2009b)] or continuous flavor symmetries (King, 2005; de Medeiros Varzielas and Ross, 2006; King and Malinsky, 2006; de Medeiros Varzielas et al., 2007b; Adulpravitchai et al., 2009b; Berger and Grossman, 2010) have also been considered [for other approaches to TB mixing, see Xing (2002), Matias and Burgess (2005), Grimus and Lavoura (2006, 2009), Luo and Xing (2006), Koide (2007a), and Babu and Gabriel (2010)], but the $A_{4}$ models have a particularly economical and attractive structure, for example, in terms of group representations and of field content. In most of the models, $A_{4}$ is accompanied by additional flavor symmetries, either discrete such as $Z_{N}$ or continuous such as $\mathrm{U}(1)$, which are necessary to eliminate unwanted couplings to ensure the needed vacuum alignment, and to reproduce the observed mass hierarchies. Given the set of flavor symmetries and having specified the field content, the nonleading corrections to $\mathrm{TB}$ mixing arising from higher order effects can be evaluated in a welldefined expansion. In the absence of specific dynamical tricks in a generic model, all the three mixing angles receive corrections of the same order of magnitude. Since the experimentally allowed departures of $\theta_{12}$ from the TB value $\sin ^{2} \theta_{12}=1 / 3$ are small, at most $\mathcal{O}\left(\lambda_{C}^{2}\right)$, with $\lambda_{C}$ as the Cabibbo angle, it follows that both $\theta_{13}$ and the deviation of $\theta_{23}$ from the maximal value are typically expected in these models to also be at most $\mathcal{O}\left(\lambda_{C}^{2}\right) .{ }^{1} \mathrm{~A}$ value of $\theta_{13} \sim \mathcal{O}\left(\lambda_{C}^{2}\right)$ is within the sensitivity of the experiments which are now in preparation and will take data in the near future.

Returning to the possibility that the agreement of the data with TB mixing is accidental, we observe that the present data do not exclude a value for $\theta_{13}$, i.e., $\theta_{13}$ $\sim \mathcal{O}\left(\lambda_{C}\right)$, larger than generally implied by models with approximate TB mixing. In fact, recent analysis of the available data lead to $\sin ^{2} \theta_{13}=0.016 \pm 0.010$ at $1 \sigma$ (Fogli et al., 2008a, 2008b), $\sin ^{2} \theta_{13}=0.010_{-0.011}^{+0.016}$ at $1 \sigma$ (Maltoni and Schwetz, 2008; Schwetz et al., 2008), $\sin ^{2} \theta_{13}$ $=0.014_{-0.011}^{+0.013}$ at $1 \sigma$ (Gonzalez-Garcia et al., 2010), and $\sin ^{2} \theta_{13}=0.010_{-0.009}^{+0.013}$ at $1 \sigma$ (Gonzalez-Garcia et al., 2010), which are compatible with both options. If experimen-

\footnotetext{
${ }^{1} \mathrm{By} \mathcal{O}\left(\lambda_{C}^{2}\right)$ we mean numerically of order $\lambda_{C}^{2}$. As $\lambda_{C} \sim 0.22 \mathrm{a}$ linear term in $\lambda_{C}$ with a small coefficient can easily be $\mathcal{O}\left(\lambda_{C}^{2}\right)$.
}

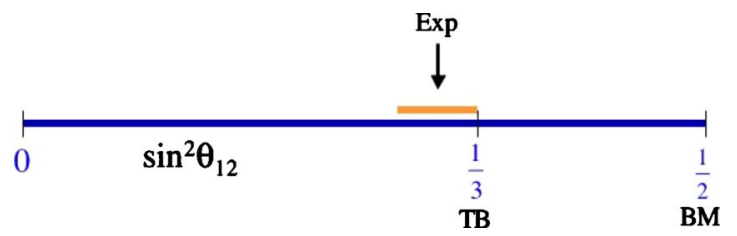

FIG. 2. (Color online) The values of $\sin ^{2} \theta_{12}$ for $\mathrm{TB}$ or $\mathrm{BM}$ mixing are compared with the data.

tally it is found that $\theta_{13}$ is near its present upper bound, this could be interpreted as an indication that the agreement with the TB mixing is accidental. In fact, a different empirical observation is that $\theta_{12}+\lambda_{C} \sim \pi / 4$, a relation known as quark-lepton complementarity (Minakata and Smirnov, 2004; Raidal, 2004), or similarly $\theta_{12}$ $+\sqrt{m_{\mu} / m_{\tau}} \sim \pi / 4$. No compelling model leading, without parameter fixing, to the exact complementarity relation has been produced so far. Probably the exact complementarity relation is to be replaced with something like $\theta_{12}+\mathcal{O}\left(\lambda_{C}\right) \sim \pi / 4$ or $\theta_{12}+\mathcal{O}\left(\sqrt{m_{\mu} / m_{\tau}}\right) \sim \pi / 4$ (which we could call "weak" complementarity) (Altarelli et al., 2004, 2009; Antusch et al., 2005; Cheung et al., 2005; Datta et al., 2005; Ferrandis and Pakvasa, 2005; Frampton and Mohapatra, 2005; Kang et al., 2005; King, 2005; Li and Ma, 2005; Lindner et al., 2005; Minakata, 2005; Ohlsson, 2005; Xing, 2005; Dighe et al., 2006; Schmidt and Smirnov, 2006; Chauhan et al., 2007; Hochmuth and Rodejohann, 2007; Plentinger et al., 2007, 2008). If we take any of these complementarity relations as a serious hint, then a scheme would be relevant where bimaximal (BM) mixing, instead of TB mixing, is the correct first approximation, modified by terms of $\mathcal{O}\left(\lambda_{C}\right)$. A comparison of the TB or BM mixing values with the data on $\sin ^{2} \theta_{12}$ is shown in Fig. 2.

A special dynamics is also needed for BM mixing and again discrete symmetry groups offer possible solutions. For example, a model (Altarelli et al., 2009) based on $S_{4}$, the permutation group of four elements, naturally leads to BM mixing in LO. This model is built in such a way that the dominant corrections to the BM mixing only arise from the charged-lepton sector at next-to-leadingorder (NLO) and naturally inherit $\lambda_{C}$ as the relevant expansion parameter. As a result, the mixing angles deviate from the $\mathrm{BM}$ values by terms $\mathcal{O}\left(\lambda_{C}\right)$ (at most), and weak complementarity holds. A crucial feature of this particular model is that only $\theta_{12}$ and $\theta_{13}$ are corrected by terms of $\mathcal{O}\left(\lambda_{C}\right)$, while $\theta_{23}$ is unchanged at this order (which is essential to make the model agree with the present data).

Other types of LO approximations for the lepton mixing pattern have been suggested. For instance, a viable first approximation of the solar mixing angle is $\theta_{12}$ $=\tan ^{-1}(1 / \varphi)$ where $\varphi=(1+\sqrt{5}) / 2$ is the golden ratio $(\mathrm{Ka}-$ jiyama et al., 2007). This leads to $\sin ^{2} \theta_{12}=1 /\left(1+\varphi^{2}\right)$ $\approx 0.276$, not far from the allowed range. Another possible connection with the golden ratio has been proposed by Rodejohann (2009). In this case, $\cos \theta_{12}=\varphi / 2$ or $\sin ^{2} \theta_{12}=1 / 4(3-\varphi) \approx 0.345$. There have been attempts 
TABLE II. Some small discrete groups used for model building.

\begin{tabular}{|c|c|c|c|c|}
\hline Group & $d$ & $\begin{array}{l}\text { Irreducible } \\
\text { representation }\end{array}$ & Presentation & References \\
\hline$D_{3} \sim S_{3}$ & 6 & $1,1^{\prime}, 2$ & $A^{3}=B^{2}=(A B)^{2}=1$ & a \\
\hline$D_{4}$ & 8 & $1_{1}, \ldots, 1_{4}, 2$ & $A^{4}=B^{2}=(A B)^{2}=1$ & $\mathrm{~b}$ \\
\hline$D_{7}$ & 14 & $1,1^{\prime}, 2,2^{\prime}, 2^{\prime \prime}$ & $A^{7}=B^{2}=(A B)^{2}=1$ & $\mathrm{c}$ \\
\hline$A_{4}$ & 12 & $1,1^{\prime}, 1^{\prime \prime}, 3$ & $A^{3}=B^{2}=(A B)^{3}=1$ & d \\
\hline$A_{5} \sim P S L_{2}(5)$ & 60 & $1,3,3^{\prime}, 4,5$ & $A^{3}=B^{2}=(B A)^{5}=1$ & $\mathrm{e}$ \\
\hline$T^{\prime}$ & 24 & $1,1^{\prime}, 1^{\prime \prime}, 2,2^{\prime}, 2^{\prime \prime}, 3$ & $A^{3}=(A B)^{3}=R^{2}=1, B^{2}=R$ & f \\
\hline$S_{4}$ & 24 & $1,1^{\prime}, 2,3,3^{\prime}$ & $\begin{array}{l}\mathrm{BM}: A^{4}=B^{2}=(A B)^{3}=1 \\
\mathrm{~TB}: A^{3}=B^{4}=\left(B A^{2}\right)^{2}=1\end{array}$ & $\mathrm{~g}$ \\
\hline$\Delta(27) \sim Z_{3} \rtimes Z_{3}$ & 27 & $1_{1}, 1_{9}, 3, \overline{3}$ & & $\mathrm{~h}$ \\
\hline$P S L_{2}(7)$ & 168 & $1,3, \overline{3}, 6,7,8$ & $A^{3}=B^{2}=(B A)^{7}=\left(B^{-1} A^{-1} B A\right)^{4}=1$ & $\mathrm{i}$ \\
\hline$T_{7} \sim Z_{7} \rtimes Z_{3}$ & 21 & $1,1^{\prime}, \overline{1^{\prime}}, 3, \overline{3}$ & $A^{7}=B^{3}=1, A B=B A^{4}$ & $\mathrm{j}$ \\
\hline
\end{tabular}

${ }^{a}$ Kubo et al., 2003; Chen et al., 2004; Kubo, 2004; Caravaglios and Morisi, 2005a, 2005b; Dermisek and Raby, 2005; Lavoura and Ma, 2005; Grimus and Lavoura, 2006; Haba and Yoshioka, 2006; Koide, 2006a, 2006b, 2007b; Mohapatra et al., 2006a, 2006b; Morisi, 2006; Morisi and Picariello, 2006; Tanimoto and Yanagida, 2006; Teshima, 2006; Kaneko et al., 2007; Chen and Wolfenstein, 2008; Feruglio and Lin, 2008; and Picariello, 2008.

${ }^{\mathrm{b}}$ Grimus et al., 2004 and Adulpravitchai, Blum, and Hagedorn, 2009.

${ }^{c}$ Blum, Hagedorn, and Hohenegger, 2008 and Blum, Hagedorn, and Lindner, 2008.

${ }^{\mathrm{d}} \mathrm{Ma}$ and Rajasekaran, 2001; Babu et al., 2003; Hirsch et al., 2004, 2005, 2007, 2008; Ma, 2004a, 2004b, 2005a, 2005b, 2005c, 2006b, 2006c, 2007; Altarelli and Feruglio, 2005, 2006; Babu and He, 2005; Chen et al., 2005; Zee, 2005; Adhikary et al., 2006; He et al., 2006; Ma et al., 2006; Altarelli et al., 2007, 2008; Lavoura and Kuhbock, 2007; Morisi et al., 2007; Yin, 2007; Adhikary and Ghosal, 2008; Bazzocchi, Frigerio, and Morisi, 2008; Bazzocchi, Kaneko, and Morisi, 2008; Bazzocchi, Morisi, and Picariello, 2008; Brahmachari et al., 2008; Csaki et al., 2008; Frampton and Matsuzaki, 2008; Grimus and Kuhbock, 2008; Honda and Tanimoto, 2008; Altarelli and Meloni, 2009; Bazzocchi, Morisi, Picariello, and Torrente-Lujan, 2009; Ciafaloni et al., 2009; Lin, 2009a, 2009b; Morisi, 2009; Antusch et al., 2010; del Aguila et al., 2010; and Kadosh and Pallante, 2010.

${ }^{\mathrm{e}}$ Everett and Stuart, 2009.

${ }^{\mathrm{f}}$ Frampton and Kephart, 1995, 2007; Aranda et al., 2000a, 2000b; Aranda, 2007; Carr and Frampton, 2007; Chen and Mahanthappa, 2007; Feruglio et al., 2007; Ding, 2008; and Frampton and Matsuzaki, 2009.

${ }^{\mathrm{g}}$ Mohapatra et al., 2004; Cai and Yu, 2006; Hagedorn et al., 2006, 2010; Ma, 2006a; Zhang, 2007; Bazzocchi and Morisi, 2009; Bazzocchi et al., 2009a, 2009b; Dutta et al., 2009, 2010; Ishimori et al., 2009, 2010; Ding, 2010; Meloni, 2010; and Morisi and Peinado, 2010.

${ }^{\mathrm{h}}$ de Medeiros Varzielas et al., 2007a; Luhn et al., 2007b; Grimus and Lavoura, 2008; Ma, 2008; and Bazzocchi and de Medeiros Varzielas, 2009.

${ }^{\mathrm{i}}$ Luhn et al., 2007a and King and Luhn, 2009a, 2009b.

${ }^{\mathrm{j}}$ Luhn et al., 2007c.

to reproduce these values by exploiting flavor symmetries of icosahedral type (Everett and Stuart, 2009) for the first possibility or of dihedral type (Adulpravitchai, Blum, and Rodejohann, 2009) for the second case.

Thus, discrete flavor symmetries may play an important role in models of neutrino mixing. In particular, this is the case if some special patterns indicated by the data as possible first approximations, such as TB or BM mixing or others, are indeed physically relevant. A list of the simplest discrete groups that have been considered for neutrino mixing, with some of their properties, is given in Table II. In the present review, we discuss the formalism and the physics of a nonexhaustive list of models of neutrino mixing based on discrete symmetries.

\section{SPECIAL PATTERNS OF NEUTRINO MIXING}

Given the Pontecorvo-Maki-Nagakawa-Sakata (PMNS) mixing matrix $U$ [see Altarelli and Feruglio (2004) for its general definition and parametrization], the general form of the neutrino mass matrix, in terms of the (complex ${ }^{2}$ ) mass eigenvalues $m_{1}, m_{2}, m_{3}$, in the basis where charged leptons are diagonal, is given by

\footnotetext{
${ }^{2}$ We absorb the Majorana phases in the mass eigenvalues $m_{i}$ rather than in the mixing matrix $U$. The dependence on these phases drops in neutrino oscillations.
} 


$$
m_{\nu}=U^{*} \operatorname{diag}\left(m_{1}, m_{2}, m_{3}\right) U^{\dagger} .
$$

We present here a number of particularly relevant forms of $U$ and $m_{\nu}$ that will be important in the following. We start with the most general mass matrix that corresponds to $\theta_{13}=0$ and $\theta_{23}$ maximal, that is, to $U$ given by (in a particular phase convention)

$$
U=\left(\begin{array}{ccc}
c_{12} & s_{12} & 0 \\
-\frac{s_{12}}{\sqrt{2}} & \frac{c_{12}}{\sqrt{2}} & -\frac{1}{\sqrt{2}} \\
-\frac{s_{12}}{\sqrt{2}} & \frac{c_{12}}{\sqrt{2}} & \frac{1}{\sqrt{2}}
\end{array}\right),
$$

with $c_{12} \equiv \cos \theta_{12}$ and $s_{12} \equiv \sin \theta_{12}$. By applying Eq. (5) we obtain a matrix of the form (Fukuyama and Nishiura, 1997; Mohapatra and Nussinov, 1999; Lam, 2001; Ma and Raidal, 2001; Ghosal, 2003; Kitabayashi and Yasue, 2003, 2005; de Gouvêa, 2004; Grimus and Lavoura, 2004; Koide, 2004; Grimus et al., 2005; Mohapatra and Nasri, 2005; Mohapatra and Rodejohann, 2005; Mohapatra et al., 2005a, 2005b; Ahn et al., 2006)

$$
m=\left(\begin{array}{lll}
x & y & y \\
y & z & w \\
y & w & z
\end{array}\right),
$$

with complex coefficients $x, y, z$, and $w$. This matrix is the most general one that is symmetric under 2-3 (or $\mu-\tau)$ exchange or

$$
m_{\nu}=A_{23} m_{\nu} A_{23},
$$

where $A_{23}$ is given by

$$
A_{23}=\left(\begin{array}{lll}
1 & 0 & 0 \\
0 & 0 & 1 \\
0 & 1 & 0
\end{array}\right) .
$$

The solar mixing angle $\theta_{12}$ is given by

$$
\begin{aligned}
\sin ^{2} 2 \theta_{12} & =\frac{8\left|x^{*} y+y^{*}(w+z)\right|^{2}}{8\left|x^{*} y+y^{*}(w+z)\right|^{2}+\left(|w+z|^{2}-|x|^{2}\right)^{2}} \\
& =\frac{8 y^{2}}{(x-w-z)^{2}+8 y^{2}},
\end{aligned}
$$

where the second equality applies to real parameters. Since $\theta_{13}=0$ there is no $C P$ violation in neutrino oscillations, and the only physical phases are the Majorana ones, accounted for by the general case of complex parameters. Here we restrict our consideration to real parameters. There are four of them in Eq. (7) which correspond to the three mass eigenvalues and one remaining mixing angle $\theta_{12}$. Models with $\mu$ - $\tau$ symmetry have been extensively studied (Fukuyama and Nishiura, 1997; Mohapatra and Nussinov, 1999; Lam, 2001; Ma and Raidal, 2001; Ghosal, 2003; Kitabayashi and Yasue, 2003, 2005; de Gouvêa, 2004; Grimus and Lavoura, 2004; Koide, 2004; Grimus et al., 2005; Mohapatra and Nasri, 2005; Mohapatra and Rodejohann, 2005; Mohapatra et al., 2005a, 2005b; Ahn et al., 2006; Ge et al., 2010).
The particularly important case of TB mixing is obtained when $\sin ^{2} 2 \theta_{12}=8 / 9$ or $x+y=w+z .^{3}$ In this case, the matrix $m_{\nu}$ takes the form

$$
m_{\nu}=\left(\begin{array}{ccc}
x & y & y \\
y & x+v & y-v \\
y & y-v & x+v
\end{array}\right) .
$$

In fact, in this case $U=U_{\mathrm{TB}}$ is given by (Harrison and Scott, 2002, 2003, 2004; Harrison et al., 2002)

$$
U_{\mathrm{TB}}=\left(\begin{array}{ccc}
\sqrt{\frac{2}{3}} & \frac{1}{\sqrt{3}} & 0 \\
-\frac{1}{\sqrt{6}} & \frac{1}{\sqrt{3}} & -\frac{1}{\sqrt{2}} \\
-\frac{1}{\sqrt{6}} & \frac{1}{\sqrt{3}} & \frac{1}{\sqrt{2}}
\end{array}\right),
$$

and from Eq. (5) one obtains

$$
m_{\nu}=m_{1} \Phi_{1} \Phi_{1}^{T}+m_{2} \Phi_{2} \Phi_{2}^{T}+m_{3} \Phi_{3} \Phi_{3}^{T},
$$

where

$$
\begin{aligned}
& \Phi_{1}^{T}=\frac{1}{\sqrt{6}}(2,-1,-1), \quad \Phi_{2}^{T}=\frac{1}{\sqrt{3}}(1,1,1), \\
& \Phi_{3}^{T}=\frac{1}{\sqrt{2}}(0,-1,1)
\end{aligned}
$$

are the respective columns of $U_{\mathrm{TB}}$ and $m_{i}$ are the neutrino mass eigenvalues $\left(m_{1}=x-y, m_{2}=x+2 y\right.$, and $m_{3}=x$ $-y+2 v)$. It is easy to see that the TB mass matrix in Eqs. (13) and (14) is indeed of the form in Eq. (11). All patterns for the neutrino spectrum are, in principle, possible. For a hierarchical spectrum $m_{3} \gg m_{2} \gg m_{1}, m_{3}^{2}$ $\sim \Delta m_{\mathrm{atm}}^{2}, m_{2}^{2} / m_{3}^{2} \sim \Delta m_{\mathrm{sol}}^{2} / \Delta m_{\mathrm{atm}}^{2}$, and $m_{1}$ could be negligible. However, degenerate masses and inverse hierarchy can also be reproduced: for example, by taking $m_{3}$ $=-m_{2}=m_{1}$ we have a degenerate model, while for $m_{1}$ $=-m_{2}$ and $m_{3}=0$ an inverse hierarchy case is realized (stability under renormalization group running [for a review see, for example, Chankowski and Pokorski (2002)] strongly prefers opposite signs for the first and second eigenvalues which are related to solar oscillations and have the smallest mass squared splitting).

Note that the mass matrix for TB mixing, in the basis where charged leptons are diagonal, as given in Eq. (11), can be specified as the most general matrix which is invariant under $\mu-\tau$ (or 2-3) symmetry [see Eqs. (8) and (9)] and, in addition, under the action of a unitary symmetric matrix $S_{\mathrm{TB}}$ (actually $S_{\mathrm{TB}}^{2}=1$ and $\left[S_{\mathrm{TB}}, A_{23}\right]=0$ ),

$$
m_{\nu}=S_{\mathrm{TB}} m_{\nu} S_{\mathrm{TB}}, \quad m_{\nu}=A_{23} m_{\nu} A_{23},
$$

where $S_{\mathrm{TB}}$ is given by

\footnotetext{
${ }^{3}$ The other solution $x-y=w+z$ gives rise to TB mixing in another phase convention and is physically equivalent to $x+y$ $=w+z$.
} 


$$
S_{\mathrm{TB}}=\frac{1}{3}\left(\begin{array}{ccc}
-1 & 2 & 2 \\
2 & -1 & 2 \\
2 & 2 & -1
\end{array}\right) \text {. }
$$

As a last example consider the case of BM where, in addition to $\theta_{13}=0$ and $\theta_{23}$ maximal, one also has $\sin ^{2} 2 \theta_{12}=1$. The BM mixing matrix is given by

$$
U_{\mathrm{BM}}=\left(\begin{array}{ccc}
\frac{1}{\sqrt{2}} & -\frac{1}{\sqrt{2}} & 0 \\
\frac{1}{2} & \frac{1}{2} & -\frac{1}{\sqrt{2}} \\
\frac{1}{2} & \frac{1}{2} & \frac{1}{\sqrt{2}}
\end{array}\right) .
$$

In the basis where charged-lepton masses are diagonal, from Eq. (5) we derive the effective neutrino mass matrix in the BM case,

$$
m_{\nu}=m_{1} \Phi_{1} \Phi_{1}^{T}+m_{2} \Phi_{2} \Phi_{2}^{T}+m_{3} \Phi_{3} \Phi_{3}^{T},
$$

where

$$
\begin{aligned}
& \Phi_{1}^{T}=\frac{1}{2}(\sqrt{2}, 1,1), \quad \Phi_{2}^{T}=\frac{1}{2}(-\sqrt{2}, 1,1), \\
& \Phi_{3}^{T}=\frac{1}{\sqrt{2}}(0,-1,1) .
\end{aligned}
$$

As we can see the most general mass matrix leading to $\mathrm{BM}$ mixing is of the form

$$
m_{\nu}=\left(\begin{array}{ccc}
x & y & y \\
y & z & x-z \\
y & x-z & z
\end{array}\right) .
$$

The resulting matrix can be completely characterized by the requirement of being invariant under the action of $A_{23}$ and also of the unitary, real, symmetric matrix $S_{\mathrm{BM}}$ (satisfying $S_{\mathrm{BM}}^{2}=1$ and $\left[S_{\mathrm{BM}}, A_{23}\right]=0$ ),

$$
m_{\nu}=S_{\mathrm{BM}} m_{\nu} S_{\mathrm{BM}}, \quad m_{\nu}=A_{23} m_{\nu} A_{23},
$$

with $S_{\mathrm{BM}}$ given by

$$
S_{\mathrm{BM}}=\left(\begin{array}{ccc}
0 & -\frac{1}{\sqrt{2}} & -\frac{1}{\sqrt{2}} \\
-\frac{1}{\sqrt{2}} & \frac{1}{2} & -\frac{1}{2} \\
-\frac{1}{\sqrt{2}} & -\frac{1}{2} & \frac{1}{2}
\end{array}\right) .
$$

The $m_{\nu}$ mass matrices of the previous examples were all derived in the basis where charged leptons are diagonal. It is useful to consider the product $m^{2}=m_{e}^{\dagger} m_{e}$, where $m_{e}$ is the charged-lepton mass matrix (defined as $\left.\bar{\psi}_{R} m_{e} \psi_{L}\right)$, because this product transforms as $m^{\prime 2}$ $=U_{e}^{\dagger} m^{2} U_{e}$, with $U_{e}$ as the unitary matrix that rotates the left-handed (LH) charged-lepton fields. The most general diagonal $\mathrm{m}^{2}$ is invariant under a diagonal phase matrix with three different phase factors:

$$
m_{e}^{\dagger} m_{e}=T^{\dagger} m_{e}^{\dagger} m_{e} T
$$

and, conversely, a matrix $m_{e}^{\dagger} m_{e}$ satisfying the above requirement is diagonal. If $T^{n}=1$, the matrix $T$ generates a cyclic group $Z_{n}$. In the simplest case $n=3$ and we get $Z_{3}$ but $n>3$ is equally possible. Examples are

$$
T_{\mathrm{TB}}=\left(\begin{array}{ccc}
1 & 0 & 0 \\
0 & \omega & 0 \\
0 & 0 & \omega^{2}
\end{array}\right),
$$

where $\omega^{3}=1$, so that $T_{\mathrm{TB}}^{3}=1$ or

$$
T_{\mathrm{BM}}=\left(\begin{array}{ccc}
-1 & 0 & 0 \\
0 & -i & 0 \\
0 & 0 & i
\end{array}\right),
$$

with $T_{\mathrm{BM}}^{4}=1$.

We are now in a position to explain the role of finite groups and to formulate the general strategy to obtain one of the previous special mass matrices, for example, that of TB mixing. We must find a group $G_{f}$ which, for simplicity, must be as small as possible but large enough to contain the $S$ and $T$ transformations. A limited number of products of $S$ and $T$ close a finite group $G_{f}$. Hence the group $G_{f}$ contains the subgroups $G_{S}$ and $G_{T}$ generated by monomials in $S$ and $T$, respectively. We assume that the theory is invariant under the spontaneously broken symmetry described by $G_{f}$. Then we must arrange a breaking of $G_{f}$ such that, in leading order, $G_{f}$ is broken down to $G_{S}$ in the neutrino mass sector and down to $G_{T}$ in the charged-lepton mass sector. In a good model this step must be realized in a natural way as a consequence of the stated basic principles and not put in by hand. The symmetry under $A_{23}$ in some cases is also part of $G_{f}$ (this is the case of $S_{4}$ ) and then must be preserved in the neutrino sector along with $S$ by the $G_{f}$ breaking or it could arise as a consequence of a special feature of the $G_{f}$ breaking [for example, in $A_{4}$ it is obtained by allowing only some transformation properties for the flavons with nonvanishing vacuum expectation values (VEVs)]. The explicit example of $A_{4}$ is discussed in Sec. III. Note that, along the same line, a model with $\mu-\tau$ symmetry can be realized in terms of group $S_{3}$ generated by products of $A_{23}$ and $T$ [see, for example, Feruglio and Lin (2008)].

\section{THE $A_{4}$ GROUP}

$A_{4}$ is the group of even permutations of four objects. It has $4 ! / 2=12$ elements. Geometrically, it can be seen as the invariance group of a tetrahedron (the odd permutations, for example, the exchange of two vertices, cannot be obtained by moving a rigid solid). We denote a generic permutation $(1,2,3,4) \rightarrow\left(n_{1}, n_{2}, n_{3}, n_{4}\right)$ simply by 
TABLE III. Characters of $A_{4}$.

\begin{tabular}{lcccc}
\hline \hline Class & $\chi^{1}$ & $\chi^{1^{\prime}}$ & $\chi^{1^{\prime \prime}}$ & $\chi^{3}$ \\
\hline$C_{1}$ & 1 & 1 & 1 & 3 \\
$C_{2}$ & 1 & $\omega$ & $\omega^{2}$ & 0 \\
$C_{3}$ & 1 & $\omega^{2}$ & $\omega$ & 0 \\
$C_{4}$ & 1 & 1 & 1 & -1 \\
\hline \hline
\end{tabular}

$\left(n_{1} n_{2} n_{3} n_{4}\right) . A_{4}$ can be generated by two basic permutations $S$ and $T$ given by $S=(4321)$ and $T=(2314)$. One checks immediately that

$$
S^{2}=T^{3}=(S T)^{3}=1 .
$$

This is called a "presentation" of the group. The 12 even permutations belong to four equivalence classes $(h$ and $k$ belong to the same class if there is a $g$ in the group such that $g h g^{-1}=k$ ) and are generated from $S$ and $T$ as follows:

$$
\begin{aligned}
& C_{1}: I=(1234), \\
& C_{2}: T=(2314), \quad S T=(4132), \\
& T S=(3241), \quad S T S=(1423), \\
& C_{3}: T^{2}=(3124), \quad S T^{2}=(4213), \\
& T^{2} S=(2431), \quad T S T=(1342), \\
& C_{4}: S=(4321), \quad T^{2} S T=(3412), \quad T S T^{2}=(2143) .
\end{aligned}
$$

Note that, except for the identity $I$ which always forms an equivalence class in itself, the other classes are according to the powers of $T$ (in $C_{4} S$ could as well be seen as $\left.S T^{3}\right)$.

The characters of a group $\chi_{g}^{R}$ are defined, for each element $g$, as the trace of the matrix that maps the element in a given representation $R$. From the invariance of traces under similarity transformations it follows that equivalent representations have the same characters and that characters have the same value for all elements in an equivalence class. Characters satisfy $\Sigma_{g} \chi_{g}^{R} \chi_{g}^{S *}=N \delta^{R S}$, where $N$ is the number of transformations in the group $\left(N=12\right.$ in $\left.A_{4}\right)$. For each element $h$, the character of $h$ in a direct product of representations is also the product of the characters, $\chi_{h}^{R \otimes S}=\chi_{h}^{R} \chi_{h}^{S}$, and is also equal to the sum of the characters in each representation that appears in the decomposition of $R \otimes S$. In a finite group the squared dimensions of the inequivalent irreducible representations add up to $N$. The character table of $A_{4}$ is given in Table III. From this table one derives that $A_{4}$ has four inequivalent representations: three of dimension 1, 1', and $1^{\prime \prime}$, and one of dimension 3.

It is immediate to see that the one-dimensional unitary representations are obtained by

$$
\begin{aligned}
& 1 S=1 T=1, \\
& 1^{\prime} S=1 T=e^{i 2 \pi / 3} \equiv \omega, \\
& 1^{\prime \prime} S=1 T=e^{i 4 \pi / 3} \equiv \omega^{2} .
\end{aligned}
$$

Note that $\omega=-1 / 2+i \sqrt{3} / 2$ is the cubic root of 1 and satisfies $\omega^{2}=\omega^{*}, 1+\omega+\omega^{2}=0$.

The three-dimensional unitary representation, on a basis where the element $S=S^{\prime}$ is diagonal, is built up from

$$
S^{\prime}=\left(\begin{array}{ccc}
1 & 0 & 0 \\
0 & -1 & 0 \\
0 & 0 & -1
\end{array}\right), \quad T^{\prime}=\left(\begin{array}{lll}
0 & 1 & 0 \\
0 & 0 & 1 \\
1 & 0 & 0
\end{array}\right) .
$$

The multiplication rules are as follows: the product of two 3 's gives $3 \times 3=1+1^{\prime}+1^{\prime \prime}+3+3$ and $1^{\prime} \times 1^{\prime}=1^{\prime \prime}, 1^{\prime}$ $\times 1^{\prime \prime}=1,1^{\prime \prime} \times 1^{\prime \prime}=1^{\prime}$, etc. If $3 \sim\left(a_{1}, a_{2}, a_{3}\right)$ is a triplet transforming by the matrices in Eq. (29), we have under $S^{\prime}$, $S^{\prime}\left(a_{1}, a_{2}, a_{3}\right)^{t}=\left(a_{1},-a_{2},-a_{3}\right)^{t}$ (here the upper index $t$ indicates transposition), and under $T^{\prime}, T^{\prime}\left(a_{1}, a_{2}, a_{3}\right)^{t}$ $=\left(a_{2}, a_{3}, a_{1}\right)^{t}$. Then, from two such triplets, $3_{a}$ $\sim\left(a_{1}, a_{2}, a_{3}\right)$ and $3_{b} \sim\left(b_{1}, b_{2}, b_{3}\right)$, the irreducible representations obtained from their product are

$$
\begin{aligned}
& 1=a_{1} b_{1}+a_{2} b_{2}+a_{3} b_{3}, \\
& 1^{\prime}=a_{1} b_{1}+\omega^{2} a_{2} b_{2}+\omega a_{3} b_{3}, \\
& 1^{\prime \prime}=a_{1} b_{1}+\omega a_{2} b_{2}+\omega^{2} a_{3} b_{3}, \\
& 3 \sim\left(a_{2} b_{3}, a_{3} b_{1}, a_{1} b_{2}\right), \\
& 3 \sim\left(a_{3} b_{2}, a_{1} b_{3}, a_{2} b_{1}\right) .
\end{aligned}
$$

In fact, take, for example, the expression for $1^{\prime \prime}=a_{1} b_{1}$ $+\omega a_{2} b_{2}+\omega^{2} a_{3} b_{3}$. Under $S^{\prime}$ it is invariant and under $T^{\prime}$ it goes into $a_{2} b_{2}+\omega a_{3} b_{3}+\omega^{2} a_{1} b_{1}=\omega^{2}\left[a_{1} b_{1}+\omega a_{2} b_{2}+\omega^{2} a_{3} b_{3}\right]$ which is exactly the transformation corresponding to $1^{\prime \prime}$.

In Eq. (29) we have the representation 3 on a basis where $S$ is diagonal. We shall see that for our purposes it is convenient to go to a basis where instead it is $T$ that is diagonal. This is obtained through the unitary transformations,

$$
\begin{aligned}
& T=V T^{\prime} V^{\dagger}=\left(\begin{array}{ccc}
1 & 0 & 0 \\
0 & \omega & 0 \\
0 & 0 & \omega^{2}
\end{array}\right), \\
& S=V S^{\prime} V^{\dagger}=\frac{1}{3}\left(\begin{array}{ccc}
-1 & 2 & 2 \\
2 & -1 & 2 \\
2 & 2 & -1
\end{array}\right),
\end{aligned}
$$

where 


$$
V=\frac{1}{\sqrt{3}}\left(\begin{array}{ccc}
1 & 1 & 1 \\
1 & \omega^{2} & \omega \\
1 & \omega & \omega^{2}
\end{array}\right)
$$

The matrix $V$ is special in that it is a $3 \times 3$ unitary matrix with all entries of unit absolute value. It is interesting that this matrix was proposed earlier as a possible mixing matrix for neutrinos (Cabibbo, 1978; Wolfenstein, 1978). We shall see in the following that in the $T$ diagonal basis the charged-lepton mass matrix (to be precise the matrix $\left.m_{e}^{\dagger} m_{e}\right)$ is diagonal. Note that the matrices $(S, T)$ of Eqs. (35) and (36) coincide with the matrices $\left(S_{\mathrm{TB}}, T_{\mathrm{TB}}\right)$ of Sec. II.

In this basis the product rules of two triplets, $\left(\psi_{1}, \psi_{2}, \psi_{3}\right)$ and $\left(\varphi_{1}, \varphi_{2}, \varphi_{3}\right)$ of $A_{4}$, according to the multiplication rule $3 \times 3=1+1^{\prime}+1^{\prime \prime}+3+3$ are different than in the $S$ diagonal basis [because for the Majorana mass matrices the relevant scalar product is $(a b)$ and not $\left.\left(a^{\dagger} b\right)\right]$ and are given by

$$
\begin{aligned}
& \psi_{1} \varphi_{1}+\psi_{2} \varphi_{3}+\psi_{3} \varphi_{2} \sim 1 \\
& \psi_{3} \varphi_{3}+\psi_{1} \varphi_{2}+\psi_{2} \varphi_{1} \sim 1^{\prime} \\
& \psi_{2} \varphi_{2}+\psi_{3} \varphi_{1}+\psi_{1} \varphi_{3} \sim 1^{\prime \prime}, \\
& \left(\begin{array}{l}
2 \psi_{1} \varphi_{1}-\psi_{2} \varphi_{3}-\psi_{3} \varphi_{2} \\
2 \psi_{3} \varphi_{3}-\psi_{1} \varphi_{2}-\psi_{2} \varphi_{1} \\
2 \psi_{2} \varphi_{2}-\psi_{1} \varphi_{3}-\psi_{3} \varphi_{1}
\end{array}\right) \sim 3_{S}, \quad\left(\begin{array}{l}
\psi_{2} \varphi_{3}-\psi_{3} \varphi_{2} \\
\psi_{1} \varphi_{2}-\psi_{2} \varphi_{1} \\
\psi_{3} \varphi_{1}-\psi_{1} \varphi_{3}
\end{array}\right) \sim 3_{A} .
\end{aligned}
$$

In the following, we work in the $T$ diagonal basis unless otherwise stated. In this basis the 12 matrices of the three-dimensional representation of $A_{4}$ are given by

$$
\begin{aligned}
\mathcal{C}_{1}: 1 & =\left(\begin{array}{lll}
1 & 0 & 0 \\
0 & 1 & 0 \\
0 & 0 & 1
\end{array}\right), \\
\mathcal{C}_{2}: T & =\left(\begin{array}{ccc}
1 & 0 & 0 \\
0 & \omega & 0 \\
0 & 0 & \omega^{2}
\end{array}\right), \quad S T=\frac{1}{3}\left(\begin{array}{ccc}
-1 & 2 \omega & 2 \omega^{2} \\
2 & -\omega & 2 \omega^{2} \\
2 & 2 \omega & -\omega^{2}
\end{array}\right), \\
T S & =\frac{1}{3}\left(\begin{array}{ccc}
-1 & 2 & 2 \\
2 \omega & -\omega & 2 \omega \\
2 \omega^{2} & 2 \omega^{2} & -\omega^{2}
\end{array}\right), \\
S T S & =\frac{1}{3}\left(\begin{array}{ccc}
-1 & 2 \omega^{2} & 2 \omega \\
2 \omega^{2} & -\omega & 2 \\
2 \omega & 2 & -\omega^{2}
\end{array}\right), \\
\mathcal{C}_{3}: T^{2} & =\left(\begin{array}{ccc}
1 & 0 & 0 \\
0 & \omega^{2} & 0 \\
0 & 0 & \omega
\end{array}\right), \quad S T^{2}=\frac{1}{3}\left(\begin{array}{ccc}
-1 & 2 \omega^{2} & 2 \omega \\
2 & -\omega^{2} & 2 \omega \\
2 & 2 \omega^{2} & -\omega
\end{array}\right),
\end{aligned}
$$

$$
\begin{aligned}
T^{2} S & =\frac{1}{3}\left(\begin{array}{ccc}
-1 & 2 & 2 \\
2 \omega^{2} & -\omega^{2} & 2 \omega^{2} \\
2 \omega & 2 \omega & -\omega
\end{array}\right), \\
T S T & =\frac{1}{3}\left(\begin{array}{ccc}
-1 & 2 \omega & 2 \omega^{2} \\
2 \omega & -\omega^{2} & 2 \\
2 \omega^{2} & 2 & -\omega
\end{array}\right), \\
\mathcal{C}_{4}: S= & \frac{1}{3}\left(\begin{array}{ccc}
-1 & 2 & 2 \\
2 & -1 & 2 \\
2 & 2 & -1
\end{array}\right), \\
T^{2} S T & =\frac{1}{3}\left(\begin{array}{ccc}
-1 & 2 \omega & 2 \omega^{2} \\
2 \omega^{2} & -1 & 2 \omega \\
2 \omega & 2 \omega^{2} & -1
\end{array}\right), \\
T S T^{2} & =\frac{1}{3}\left(\begin{array}{ccc}
-1 & 2 \omega^{2} & 2 \omega \\
2 \omega & -1 & 2 \omega^{2} \\
2 \omega^{2} & 2 \omega & -1
\end{array}\right) .
\end{aligned}
$$

We can now see why $A_{4}$ works for TB mixing. In Sec. II we mentioned that the most general mass matrix for TB mixing in Eq. (11), in the basis where charged leptons are diagonal, can be specified as one which is invariant under the 2-3 (or $\mu-\tau)$ symmetry and under the $S$ unitary transformation, as stated in Eq. (15) [note that $S_{\mathrm{TB}}$ in Eqs. (15) and (16) coincides with $S$ in Eq. (36)]. This observation plays a key role in leading to $A_{4}$ as a candidate group for TB mixing because $S$ is a matrix of $A_{4}$. Instead the matrix $A_{23}$ is not an element of $A_{4}$ (because the 2-3 exchange is an odd permutation). We shall see that in $A_{4}$ models the 2-3 symmetry is maintained by imposing that there are no flavons transforming as $1^{\prime}$ or 1 " that break $A_{4}$ with two different VEVs (in particular, one can assume that there are no flavons in the model transforming as $1^{\prime}$ or $\left.1^{\prime \prime}\right)$. It is also clear that a generic diagonal charged-lepton matrix $m_{e}^{\dagger} m_{e}$ is characterized by the invariance under $T$ or $T^{\dagger} m_{e}^{\dagger} m_{e} T=m_{e}^{\dagger} m_{e}$.

The group $A_{4}$ has two obvious subgroups: $G_{S}$, which is a reflection subgroup generated by $S$, and $G_{T}$, which is the group generated by $T$, isomorphic to $Z_{3}$. If the flavor symmetry associated to $A_{4}$ is broken by the VEV of a triplet $\varphi=\left(\varphi_{1}, \varphi_{2}, \varphi_{3}\right)$ of scalar fields, there are two interesting breaking patterns. The VEV

$$
\langle\varphi\rangle=\left(v_{S}, v_{S}, v_{S}\right)
$$

breaks $A_{4}$ down to $G_{S}$, while

$$
\langle\varphi\rangle=\left(v_{T}, 0,0\right)
$$

breaks $A_{4}$ down to $G_{T}$. As we will see, $G_{S}$ and $G_{T}$ are the relevant low-energy symmetries of the neutrino and the charged-lepton sectors, respectively. Indeed, we have already seen that the TB mass matrix is invariant under $G_{S}$ and a diagonal charged-lepton mass $m_{e}^{\dagger} m_{e}$ is invariant under $G_{T}$. 
TABLE IV. Transformation properties of all the fields.

\begin{tabular}{lccccccccccccc}
\hline \hline & $l$ & $e^{c}$ & $\mu^{c}$ & $\tau^{c}$ & $\nu^{c}$ & $h_{u, d}$ & $\theta$ & $\varphi_{T}$ & $\varphi_{S}$ & $\xi$ & $\varphi_{0}^{T}$ & $\varphi_{0}^{S}$ & $\xi_{0}$ \\
\hline$A_{4}$ & 3 & 1 & $1^{\prime \prime}$ & $1^{\prime}$ & 3 & 1 & 1 & 3 & 3 & 1 & 3 & 3 & 1 \\
$Z_{3}$ & $\omega$ & $\omega^{2}$ & $\omega^{2}$ & $\omega^{2}$ & $\omega^{2}$ & 1 & 1 & 1 & $\omega^{2}$ & $\omega^{2}$ & 1 & $\omega^{2}$ & $\omega^{2}$ \\
$\mathrm{U}(1)_{\mathrm{FN}}$ & 0 & 4 & 2 & 0 & 0 & 0 & -1 & 0 & 0 & 0 & 0 & 0 & 0 \\
$\mathrm{U}(1)_{R}$ & 1 & 1 & 1 & 1 & 1 & 0 & 0 & 0 & 0 & 0 & 2 & 2 \\
\hline \hline
\end{tabular}

\section{APPLYING $A_{4}$ TO LEPTON MASSES AND MIXINGS}

In the lepton sector a typical $A_{4}$ model works as follows (Altarelli and Feruglio, 2006). One assigns leptons to the four inequivalent representations of $A_{4}$ : LH lepton doublets $l$ transform as a triplet 3 , while the $\mathrm{RH}$ charged leptons $e^{c}, \mu^{c}$, and $\tau^{c}$ transform as $1,1^{\prime \prime}$, and $1^{\prime}$, respectively. Here we consider a seesaw realization, so we also introduce conjugate neutrino fields $\nu^{c}$ transforming as a triplet of $A_{4}$. We adopt a supersymmetric (SUSY) context also to make contact with grand unification [flavor symmetries are supposed to act near the grand unified theory (GUT) scale]. In fact, as is well known, SUSY is important in GUTs for offering a solution to the hierarchy problem, for improving coupling unification, and for making the theory compatible with bounds on proton decay. However, in models of lepton mixing SUSY also helps in obtaining the vacuum alignment because the SUSY constraints are very strong and limit the form of the superpotential. Thus, SUSY is not necessary but it is a plausible and useful ingredient. The flavor symmetry is broken by two triplets $\varphi_{S}$ and $\varphi_{T}$ and by one or more singlets $\xi$. All these fields are invariant under the standard model (SM) gauge symmetry. Two Higgs doublets $h_{u, d}$, invariant under $A_{4}$, are also introduced. One can obtain the observed hierarchy among $m_{e}, m_{\mu}$, and $m_{\tau}$ by introducing an additional $\mathrm{U}(1)_{\mathrm{FN}}$ flavor symmetry (Froggatt and Nielsen, 1979) under which only the RH lepton sector is charged [recently some models were proposed with a different VEV alignment such that the charged-lepton hierarchies are obtained without introducing a U(1) symmetry (Altarelli and Meloni, 2009; Lin, 2009a, 2009b)]. We recall that U(1) FN $_{\mathrm{FN}}$ is a simplest flavor symmetry where particles in different generations are assigned, in general, different values of an Abelian charge. Also Higgs fields may get a nonzero charge. When the symmetry is spontaneously broken the entries of mass matrices are suppressed if there is a charge mismatch and more so if the corresponding mismatch is larger. We assign the Froggatt-Nielsen (FN) charges $0, q$, and $2 q$ to $\tau^{c}, \mu^{c}$, and $e^{c}$, respectively. There is some freedom in the choice of $q$. Here we take $q=2$. By assuming that a flavon $\theta$, carrying a negative unit of FN charge, acquires a VEV $\langle\theta\rangle / \Lambda \equiv \lambda<1$, the Yukawa couplings become field dependent quantities $y_{e, \mu, \tau}$ $=y_{e, \mu, \tau}(\theta)$ and we have

$$
y_{\tau} \approx \mathcal{O}(1), \quad y_{\mu} \approx O\left(\lambda^{2}\right), \quad y_{e} \approx O\left(\lambda^{4}\right) .
$$

Had we chosen $q=1$, we would have needed $\langle\theta\rangle / \Lambda$ of order $\lambda^{2}$ to reproduce the above result. The superpotential term for lepton masses $w_{l}$ is given by

$$
\begin{aligned}
w_{l}= & y_{e} e^{c}\left(\varphi_{T} l\right)+y_{\mu} \mu^{c}\left(\varphi_{T} l\right)^{\prime}+y_{\tau} \tau^{c}\left(\varphi_{T} l\right)^{\prime \prime}+y\left(\nu^{c} l\right) \\
& +\left(x_{A} \xi+\tilde{x}_{A} \tilde{\xi}\right)\left(\nu^{c} \nu^{c}\right)+x_{B}\left(\varphi_{S} \nu^{c} \nu^{c}\right)+\text { H.c. }+\cdots,
\end{aligned}
$$

with dots denoting higher-dimensional operators that lead to corrections to the LO approximation. In our notation, the product of two triplets (33) transforms as 1, (33)' transforms as $1^{\prime}$, and (33)" transforms as $1^{\prime \prime}$. To keep our formulas compact, we omit writing the Higgs and flavon fields $h_{u, d}, \theta$, and the cutoff scale $\Lambda$. For instance, $y_{e} e^{c}\left(\varphi_{T} l\right)$ stands for $y_{e} e^{c}\left(\varphi_{T} l\right) h_{d} \theta^{4} / \Lambda^{5}$. The parameters of the superpotential $w_{l}$ are complex, in particular those responsible for the heavy neutrino Majorana masses, $x_{A, B}$. Some terms allowed by the $A_{4}$ symmetry, such as the terms obtained by the exchange $\varphi_{T} \leftrightarrow \varphi_{S}$ [or the term $\left(\nu^{c} \nu^{c}\right)$ ], are missing in $w_{l}$. Their absence is crucial and in each version of $A_{4}$ models is motivated by additional symmetries. [Altarelli and Feruglio (2005) discussed a natural solution of this problem based on a formulation with extra dimensions; for a similar approach see also Kadosh and Pallante (2010).] In the present version the additional symmetry is $Z_{3}$. A U $(1)_{R}$ symmetry related to $R$ parity and the presence of driving fields in the flavon superpotential are common features of supersymmetric formulations. Eventually, after the inclusion of $N=1$ SUSY breaking effects, the $\mathrm{U}(1)_{R}$ symmetry will be broken at the low-energy scale $m_{\text {SUSY }}$ down to the discrete $R$ parity. Supersymmetry also helps producing and maintaining the hierarchy $\left\langle h_{u, d}\right\rangle=v_{u, d}$ $\ll \Lambda$, where $\Lambda$ is the cutoff scale of the theory. The fields in the model and their classification under the symmetry are summarized in Table IV.

In this setup it can be shown that the fields $\varphi_{T}, \varphi_{S}$, and $\xi$ develop a VEV along the directions

$$
\begin{aligned}
& \left\langle\varphi_{T}\right\rangle=\left(v_{T}, 0,0\right), \\
& \left\langle\varphi_{S}\right\rangle=\left(v_{S}, v_{S}, v_{S}\right), \\
& \langle\xi\rangle=u .
\end{aligned}
$$

A crucial part of all serious $A_{4}$ models is the dynamical generation of this alignment in a natural way. See Altarelli and Feruglio (2006) for a proof that the above alignment naturally follows from the most general LO superpotential implied by the symmetries of the model. As mentioned, the group $A_{4}$ has two obvious subgroups: $G_{S}$, which is a reflection subgroup generated by $S$, and $G_{T}$, which is the group generated by $T$, isomorphic to $Z_{3}$. In the basis where $S$ and $T$ are given by Eq. (16), the 
$\operatorname{VEV}\left\langle\varphi_{T}\right\rangle=\left(v_{T}, 0,0\right)$ breaks $A_{4}$ down to $G_{T}$, while $\left\langle\varphi_{S}\right\rangle$ $=\left(v_{S}, v_{S}, v_{S}\right)$ breaks $A_{4}$ down to $G_{S}$.

If the alignment in Eq. (43) is realized, at the leading order of the $1 / \Lambda$ expansion, the mass matrices $m_{l}$ and $m_{\nu}$ for charged leptons and neutrinos correspond to TB mixing. The charged-lepton mass matrix is diagonal,

$$
m_{l}=v_{d} \frac{v_{T}}{\Lambda}\left(\begin{array}{ccc}
y_{e} & 0 & 0 \\
0 & y_{\mu} & 0 \\
0 & 0 & y_{\tau}
\end{array}\right) .
$$

The charged fermion masses are given by

$$
m_{e}=y_{e} v_{d} \frac{v_{T}}{\Lambda}, \quad m_{\mu}=y_{\mu} v_{d} \frac{v_{T}}{\Lambda}, \quad m_{\tau}=y_{\tau} v_{d} \frac{v_{T}}{\Lambda},
$$

where the suppression coming from the breaking of $\mathrm{U}(1)_{F N}$ is understood. For example, $y_{e}$ stands for $y_{e} \theta^{4} / \Lambda^{4}$. In the neutrino sector, after electroweak and $A_{4}$ symmetry breaking we have the Dirac and Majorana masses,

$$
\begin{aligned}
& m_{\nu}^{D}=\left(\begin{array}{lll}
1 & 0 & 0 \\
0 & 0 & 1 \\
0 & 1 & 0
\end{array}\right) y v_{u}, \\
& M=\left(\begin{array}{ccc}
A+2 B / 3 & -B / 3 & -B / 3 \\
-B / 3 & 2 B / 3 & A-B / 3 \\
-B / 3 & A-B / 3 & 2 B / 3
\end{array}\right) u,
\end{aligned}
$$

where

$$
A \equiv 2 x_{A}, \quad B \equiv 2 x_{B} \frac{v_{S}}{u} .
$$

The eigenvalues of $M$ are

$$
M_{1}=(A+B) u, \quad M_{2}=A u, \quad M_{3}=(-A+B) u .
$$

The mass matrix for light neutrinos is $m_{\nu}$ $=\left(m_{\nu}^{D}\right)^{T} M^{-1} m_{\nu}^{D}$ with eigenvalues

$$
m_{1}=\frac{y^{2} v_{u}^{2}}{M_{1}}, \quad m_{2}=\frac{y^{2} v_{u}^{2}}{M_{2}}, \quad m_{3}=\frac{y^{2} v_{u}^{2}}{M_{3}} .
$$

The mixing matrix is $U_{\mathrm{TB}}$ [Eq. (12)]. Both normal and inverted hierarchies in the neutrino mass spectrum can be realized. It is interesting that $A_{4}$ models with the seesaw mechanism typically lead to a light neutrino spectrum which satisfies the sum rule (among complex masses)

$$
\frac{1}{m_{3}}=\frac{1}{m_{1}}-\frac{2}{m_{2}} .
$$

The phases of the complex parameters $A$ and $B$ do not produce any $C P$ violation in neutrino oscillations, since $\theta_{13}=0$, but are quite important to make the above sum rule compatible with the present data on neutrino masses. A detailed discussion of a spectrum of this type has been given by Altarelli and Feruglio (2006) and Altarelli and Meloni (2009).
Both types of ordering, normal and inverted, are allowed and the above sum rule gives rise to bounds on the lightest neutrino mass. For normal ordering we have

$$
\begin{aligned}
& m_{1} \geqslant \sqrt{\frac{\Delta m_{\text {sun }}^{2}}{3}}\left(1-\frac{4 \sqrt{3}}{9} r+\cdots\right) \approx 0.004 \mathrm{eV}, \\
& m_{1} \leqslant \sqrt{\frac{\Delta m_{\text {sun }}^{2}}{3}}\left(1+\frac{4 \sqrt{3}}{9} r+\cdots\right) \approx 0.006 \mathrm{eV},
\end{aligned}
$$

and for the inverted ordering

$$
m_{3} \geqslant \sqrt{\frac{\Delta m_{\mathrm{atm}}^{2}}{8}}\left(1-\frac{1}{6} r^{2}+\cdots\right) \approx 0.017 \mathrm{eV},
$$

where the ellipses represent terms with higher powers of $r$. Note that for normal ordering the neutrino mass spectrum is essentially determined: $m_{1} \approx 0.005 \mathrm{eV}, m_{1}$ $\approx 0.01 \mathrm{eV}$, and $m_{3} \approx 0.05 \mathrm{eV}$. The possible values of $\left|m_{e e}\right|$ are also restricted. For normal hierarchy we have

$$
\left|m_{e e}\right| \approx \frac{4}{3 \sqrt{3}} \Delta m_{\mathrm{sun}}^{2} \approx 0.007 \mathrm{eV},
$$

while for inverted hierarchy

$$
\left|m_{e e}\right| \geqslant \sqrt{\frac{\Delta m_{\mathrm{atm}}^{2}}{8}} \approx 0.017 \mathrm{eV}
$$

In a completely general framework, without the restrictions imposed by the flavor symmetry, $\left|m_{e e}\right|$ could vanish in the case of normal hierarchy. In this model $\left|m_{e e}\right|$ is always different from zero, though its value for normal hierarchy is probably too small to be detected in the next generation of $0 \nu \beta \beta$ experiments.

Note that in the charged-lepton sector the flavor symmetry $A_{4}$ is broken by $\left\langle\varphi_{T}\right\rangle$ down to $G_{T}$. Actually the above mass terms for charged leptons are the most general allowed by the symmetry $G_{T}$. At leading order in $1 / \Lambda$, charged-lepton masses are diagonal simply because there is a low-energy $G_{T}$ symmetry. In the neutrino sector $A_{4}$ is broken down to $G_{S}$, though neutrino masses in this model are not the most general ones allowed by $G_{S}$. The additional property which is needed, the invariance under $A_{\mu \tau}$, is obtained by stipulating that there are no $A_{4}$ breaking flavons transforming as $1^{\prime}$ and $1^{\prime \prime}$. In fact, from Eq. (38), we see that the expressions for (33)' and (33)" are not 2-3 symmetric.

At the next level of approximation each term of the superpotential is corrected by operators of higher dimension whose contributions are suppressed by at least one power of VEVs in units of $\Lambda$. The corrections to the relevant part of the superpotential determine small deviations from the LO VEV alignment configuration. The NLO corrections to mass and mixing matrices are obtained by inserting the corrected VEV alignment in the $\mathrm{LO}$ operators plus the contribution of the new operators evaluated with the unperturbed VEVs. The final result is (Altarelli and Feruglio, 2006) that, when the NLO corrections are included, TB mixing is violated by small terms of the same order for all mixing angles, 


$$
\begin{aligned}
& \sin ^{2} \theta_{12}=\frac{1}{3}+\mathcal{O}(\varepsilon), \\
& \sin ^{2} \theta_{23}=\frac{1}{2}+\mathcal{O}(\varepsilon), \\
& \sin \theta_{13}=\mathcal{O}(\varepsilon),
\end{aligned}
$$

where $\varepsilon$ is of order of the typical VEV in units of $\Lambda$. The fact that TB mixing is well satisfied by the data sets the restriction $\varepsilon<\mathcal{O}\left(\lambda_{C}^{2}\right)$. From the requirement that the Yukawa coupling $y_{\tau}$ remains in the perturbative regime, we also get a lower bound on $\varepsilon$ of about 0.01 , the exact value depending on $\tan \beta=v_{u} / v_{d}$ and on the largest allowed $\left|y_{\tau}\right|$. Thus, we have

$$
0.01<\varepsilon<0.05 \text {. }
$$

From the seesaw relations in Eq. (49), assuming a coupling $y$ of order 1 , we see that the heavy RH neutrino masses are all of order $10^{15} \mathrm{GeV}$, close to the GUT scale. The cutoff of the theory can be estimated form Eq. (56) to be close to $10^{17} \mathrm{GeV}$.

The above results in Eqs. (44)-(54) on the lepton mass matrices and the neutrino spectrum refer to the LO approximation. Relations among neutrino masses can be affected by NLO corrections but, for $\varepsilon$ varying in the range of Eq. (56), the bounds (51) and (52) do not appreciably change [see, for example, Barry and Rodejohann (2010) for a numerical study of the deviations induced by vacuum misalignment]. Also corrections induced by the renormalization group evolution of the parameters can modify the above predictions but only in the case of sufficiently degenerate mass levels $m_{1}$ and $m_{2}$ with equal phases, which occurs for inverted mass ordering and far from the lower bound (52) (Lin et al., 2010). The expansion parameter $\varepsilon$ directly controls other observables, such as the $C P$ asymmetries of leptogenesis and the rates of lepton flavor violating transitions. This provides an interesting link between the physics in the early Universe relevant for leptogenesis and the lowenergy physics accessible in current experiments. We discuss the interplay between discrete flavor symmetries and leptogenesis in Sec. X.

\section{POSSIBLE ORIGIN OF $A_{4}$}

There is an interesting relation (Altarelli and Feruglio, 2006) between the $A_{4}$ model considered so far and the modular group. This relation could possibly be relevant to understand the origin of the $A_{4}$ symmetry from a more fundamental layer of the theory. The modular group $\Gamma$ is the group of linear fractional transformations acting on a complex variable $z$,

$$
z \rightarrow \frac{a z+b}{c z+d}, \quad a d-b c=1,
$$

where $a, b, c$, and $d$ are integers. There are infinite elements in $\Gamma$, but all of them can be generated by the two transformations,

$$
s: z \rightarrow-\frac{1}{z}, \quad t: z \rightarrow z+1 .
$$

The transformations $s$ and $t$ in Eq. (58) satisfy the relations

$$
s^{2}=(s t)^{3}=1,
$$

and, conversely, these relations provide an abstract characterization of the modular group. Since relation (26) is a particular case of more general constraint (59), it is clear that $A_{4}$ is a very small subgroup of the modular group and that the $A_{4}$ representations discussed above are also representations of the modular group. In string theory the transformations (58) operate in many different contexts. For instance, the role of the complex variable $z$ can be played by a field, whose VEV can be related to a physical quantity such as a compactification radius or a coupling constant. In that case $s$ in Eq. (58) represents a duality transformation and $t$ in Eq. (58) represents the transformation associated to an "axionic" symmetry.

A different way to understand the dynamical origin of $A_{4}$ was recently presented by Altarelli et al. (2007) where it is shown that the $A_{4}$ symmetry can be obtained by orbifolding starting from a model in six dimensions. In this approach $A_{4}$ appears as the remnant of the reduction from six-dimensional (6D) to four-dimensional (4D) space-time symmetry induced by the special orbifolding adopted. This approach suggests a deep relation between flavor symmetry in four dimensions and spacetime symmetry in extra dimensions.

The orbifolding is defined as follows. We consider a quantum field theory in six dimensions, with two extra dimensions compactified on an orbifold $T^{2} / Z_{2}$. We denote by $z=x_{5}+i x_{6}$ the complex coordinate describing the extra space. The torus $T^{2}$ is defined by identifying in the complex plane the points related by

$$
z \rightarrow z+1, \quad z \rightarrow z+\gamma, \quad \gamma=e^{i(\pi / 3)},
$$

where our length unit $2 \pi R$ has been set to 1 for the time being. The parity $Z_{2}$ is defined by

$$
z \rightarrow-z
$$

and the orbifold $T^{2} / Z_{2}$ can be represented by the fundamental region given by the triangle with vertices 0,1 , and $\gamma$ (see Fig. 3). The orbifold has four fixed points, $\left(z_{1}, z_{2}, z_{3}, z_{4}\right)=(1 / 2,(1+\gamma) / 2, \gamma / 2,0)$. The fixed point $z_{4}$ is also represented by the vertices 1 and $\gamma$. In the orbifold, the segments labeled by $a$ in Fig. 1, $(0,1 / 2)$ and $(1,1 / 2)$, are identified and similarly for those labeled by $b,(1,(1+\gamma) / 2)$ and $(\gamma,(1+\gamma) / 2)$, and those labeled by $c$, $(0, \gamma / 2)$ and $(\gamma, \gamma / 2)$. Therefore, the orbifold is a regular tetrahedron with vertices at the four fixed points.

The symmetry of the uncompactified 6D space-time is broken by compactification. Here we assume that, before compactification, the space-time symmetry coincides with the product of $6 \mathrm{D}$ translations and $6 \mathrm{D}$ proper Lorentz transformations. The compactification breaks part of this symmetry. However, due to the special ge- 


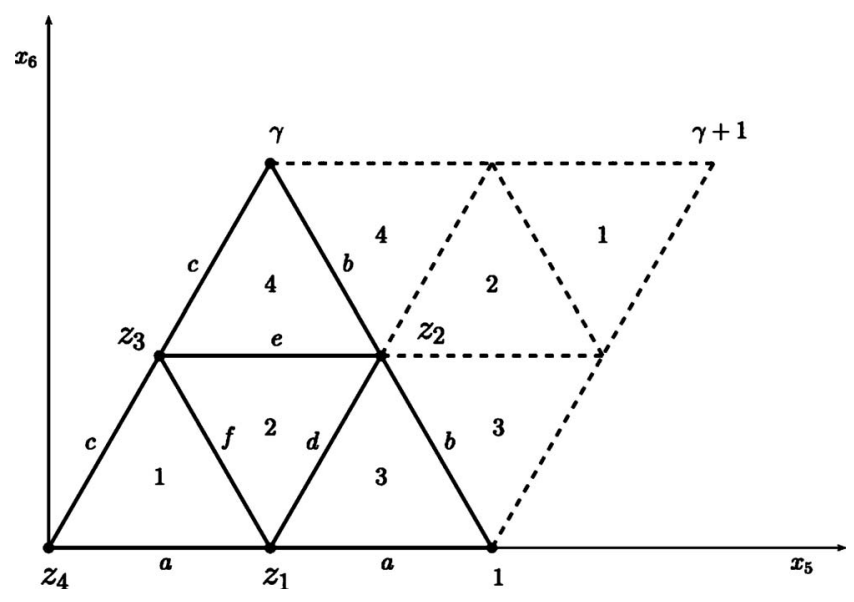

FIG. 3. Orbifold $T_{2} / Z_{2}$. The regions with the same numbers are identified with each other. The four triangles bounded by solid lines form the fundamental region, where the edges with the same letters are also identified. The orbifold $T_{2} / Z_{2}$ is exactly a regular tetrahedron with six edges $(a, b, c, d, e$, and $f)$ and four vertices $\left(z_{1}, z_{2}, z_{3}\right.$, and $\left.z_{4}\right)$, corresponding to the four fixed points of the orbifold.

ometry of our orbifold, a discrete subgroup of rotations and translations in the extra space is left unbroken. This group can be generated by two transformations,

$$
\mathcal{S}: z \rightarrow z+\frac{1}{2}, \quad \mathcal{T}: z \rightarrow \omega z \quad \omega \equiv \gamma^{2} .
$$

Indeed, $\mathcal{S}$ and $\mathcal{T}$ induce even permutations of the four fixed points,

$$
\begin{aligned}
& \mathcal{S}:\left(z_{1}, z_{2}, z_{3}, z_{4}\right) \rightarrow\left(z_{4}, z_{3}, z_{2}, z_{1}\right), \\
& \mathcal{T}:\left(z_{1}, z_{2}, z_{3}, z_{4}\right) \rightarrow\left(z_{2}, z_{3}, z_{1}, z_{4}\right),
\end{aligned}
$$

thus generating the group $A_{4}$. From the previous equations we immediately verify that $\mathcal{S}$ and $\mathcal{T}$ satisfy the characteristic relations obeyed by the generators of $A_{4}: \mathcal{S}^{2}$ $=\mathcal{T}^{3}=(\mathcal{S T})^{3}=1$. These relations are actually satisfied not only at the fixed points but on the whole orbifold, as can be easily checked from the general definitions of $\mathcal{S}$ and $\mathcal{T}$ in Eq. (62), with the help of the orbifold defining rules in Eqs. (60) and (61).

We can exploit this particular geometry of the internal space to build a model with $A_{4}$ flavor symmetry. There are $4 \mathrm{D}$ branes at the four fixed points of the orbifolding and the tetrahedral symmetry of $A_{4}$ connects these branes. The standard model fields have components on the fixed point branes, while the scalar fields necessary for the $A_{4}$ breaking are in the bulk. Each brane field, either a triplet or a singlet, has components on all of the four fixed points (in particular, all components are equal for a singlet) but the interactions are local; i.e., all vertices involve products of field components at the same space-time point. In the low-energy limit this model coincides with the one illustrated in Sec. IV. Unfortunately in such a limit the $6 \mathrm{D}$ construction does not provide additional constraints or predictions.

This construction can be embedded in a SU(5) GUT (Burrows and King, 2010). Other discrete groups can arise from the compactification of two extra dimensions on orbifolds and the possibilities have been classified by Adulpravitchai et al. (2009a) within a field theory approach. In string theory the flavor symmetry can be larger than the isometry of the compact space. For instance, in heterotic orbifold models the orbifold geometry combines with the space group selection rules of the string, as shown by Kobayashi et al. (2007). Discrete flavor symmetries from magnetized or intersecting D branes are discussed in Abe et al. (2009). Discrete symmetries can also arise from the spontaneous breaking of continuous ones. Such a possibility has been discussed by Adulpravitchai et al. (2009b) and Berger and Grossman (2010).

\section{ALTERNATIVE ROUTES TO TB MIXING}

While $A_{4}$ is the minimal flavor group leading to TB mixing, alternative flavor groups have been studied and can lead to interesting variants with some specific features.

Recently, Lam (2008), claimed that, in order to obtain the TB mixing "without fine tuning," the finite group must be $S_{4}$ or a larger group containing $S_{4}$. We believe this claim is not well grounded being based on an abstract mathematical criterium for a natural model [see also Grimus et al. (2009)]. For us a physical field theory model is natural if the interesting results are obtained from the most general Lagrangian compatible with the stated symmetry and the specified representation content for the flavons. For example, we obtain from $A_{4}$ (which is a subgroup of $S_{4}$ ) a natural, in our sense, model for the TB mixing by simply not including symmetry breaking flavons transforming like the $1^{\prime}$ and the 1 " representations of $A_{4}$. This limitation on the transformation properties of the flavons is not allowed by the rules specified in Lam (2008) which demand that the symmetry breaking is induced by all possible kinds of flavons (note that, according to this criterium, the SM of electroweak interactions would not be natural because only the Higgs doublets are introduced). Rather for naturalness we also require that additional physical properties, such as the VEV alignment or the hierarchy of chargedlepton masses also follow from the assumed symmetry and are not obtained by fine-tuning parameters: for this actually $A_{4}$ can be more effective than $S_{4}$ because it possesses three different singlet representations, $1,1^{\prime}$, and $1^{\prime \prime}$.

Models of neutrino mixing based on $S_{4}$ have, in fact, been studied (Mohapatra et al., 2004; Cai and Yu, 2006; Hagedorn et al., 2006, 2010; Ma, 2006a; Bazzocchi and Morisi, 2009; Bazzocchi et al., 2009a, 2009b; Dutta et al., 2009, 2010; Ishimori et al., 2009, 2010; Ding, 2010; Meloni, 2010; Morisi and Peinado, 2010). The group of the permutations of four objects $S_{4}$ has 24 elements and 5 equivalence classes (the character table is given in Table V) that correspond to five inequivalent irreducible representations: two singlets, one doublet, two triplets: $1_{1}, 1_{2}, 2,3_{1}$, and $3_{2}$ (see Table II). Note that the squares 
TABLE V. Characters of $S_{4}$.

\begin{tabular}{lccccc}
\hline \hline Class & $\chi\left(1_{1}\right)$ & $\chi\left(1_{2}\right)$ & $\chi(2)$ & $\chi\left(3_{1}\right)$ & $\chi\left(3_{2}\right)$ \\
\hline$C_{1}$ & 1 & 1 & 2 & 3 & 3 \\
$C_{2}$ & 1 & 1 & 2 & -1 & -1 \\
$C_{3}$ & 1 & -1 & 0 & 1 & -1 \\
$C_{4}$ & 1 & 1 & -1 & 0 & 0 \\
$C_{5}$ & 1 & -1 & 0 & -1 & 1 \\
\hline \hline
\end{tabular}

of the dimensions of all these representations add up to 24.

For models of TB mixing, one starts from the $S_{4}$ presentation $A^{3}=B^{4}=\left(B A^{2}\right)^{2}=1$ and identifies, up to a similarity transformation, $B^{2}=S$ and $A=T$, where $S$ and $T$ are given in Eqs. (16) and (24). In this presentation one obtains a realization of the three-dimensional representation of $S_{4}$ where $S$ and $A_{23}$ matrices in Eq. (15), leave the TB form of $m_{\nu}$ invariant in Eq. (11) as well as the matrix $T$ in Eq. (24) of invariance for $m_{e}^{\dagger} m_{e}$, all explicitly appear (Bazzocchi et al., 2009a). In $S_{4}$ the $1^{\prime}$ and $1^{\prime \prime}$ of $A_{4}$ are collected in a doublet. When the VEV of the doublet flavon is aligned along the $G_{S}$ preserving direction the resulting couplings are 2-3 symmetric as needed. In $A_{4}$ the 2-3 symmetry is only achieved if the $1^{\prime}$ and $1^{\prime \prime}$ VEVs are identical (which is the $S_{4}$ prediction). As discussed by Bazzocchi et al. (2009a), in the leptonic sector the main difference between $A_{4}$ and $S_{4}$ is that, while in the typical versions of $A_{4}$ the most general neutrino mass matrix depends on two complex parameters (related to the couplings of the singlet and triplet flavons), in $S_{4}$ it depends on three complex parameters (because the doublet is present in addition to singlet and triplet flavons).

Other flavor groups have been considered for models of TB mixing. Some of them include $S_{4}$ as a subgroup, such as $\mathrm{PSL}_{2}(7)$ (the smallest group with complex triplet representations) (Luhn et al., 2007a; King and Luhn, 2009a, 2009b), while others, such as $\Delta(27)$ [which is a discrete subgroup of SU(3)] (de Medeiros Varzielas et al., 2007a; Luhn et al., 2007b; Grimus and Lavoura, 2008; Ma, 2008; Bazzocchi and de Medeiros Varzielas, 2009) or $Z_{7} \rtimes Z_{3}$ (Luhn et al., 2007c), have no direct relation to $S_{4}$ (King, 2010). In Sec. VIII we consider $S_{4}$ again in the different contexts of BM with large corrections from the lepton sector.

A different approach to TB mixing has been proposed and developed in different versions by King and collaborators over the past few years (King, 2005, 2010; King and Malinsky, 2006; de Medeiros Varzielas and Ross, 2006; de Medeiros Varzielas et al., 2007b). The starting point is the decomposition of the neutrino mass matrix given in Eqs. (13) and (14) corresponding to exact TB mixing in the diagonal charged-lepton basis,

$$
m_{\nu}=m_{1} \Phi_{1} \Phi_{1}^{T}+m_{2} \Phi_{2} \Phi_{2}^{T}+m_{3} \Phi_{3} \Phi_{3}^{T},
$$

where $\Phi_{1}^{T}=(1 / \sqrt{6})(2,-1,-1), \quad \Phi_{2}^{T}=(1 / \sqrt{3})(1,1,1)$, and $\Phi_{3}^{T}=(1 / \sqrt{2})(0,-1,1)$ are the respective columns of $U_{\mathrm{TB}}$ and $m_{i}$ are the neutrino mass eigenvalues. Such decom- position is purely kinematical and does not possess any dynamical or symmetry content. In the King models the idea is that the three columns of $U_{\mathrm{TB}} \Phi_{i}$ are promoted to flavon fields whose VEVs break the family symmetry, with the particular vacuum alignments along the directions $\Phi_{i}$. Equation (64) directly arises in the seesaw mechanism $m_{\nu}=m_{D}^{T} M^{-1} m_{D}$ written in the diagonal $\mathrm{RH}$ neutrino mass basis $M=\operatorname{diag}\left(M_{1}, M_{2}, M_{3}\right)$, when the Dirac mass matrix is given by $m_{D}^{T}=\left(v_{1} \Phi_{1}, v_{2} \Phi_{2}, v_{3} \Phi_{3}\right)$, where $v_{i}$ are mass parameters describing the size of the VEVs. In this way, for each RH neutrino eigenvalue $M_{i}$ a particular light neutrino mass $m_{i}$ is associated. In the case of a strong neutrino hierarchy this idea can be combined with the framework of "sequential dominance," where the lightest RH neutrino with its symmetry properties fixes the heaviest light neutrino and so on. For no pronounced hierarchy the correspondence between $M_{i}$ and $m_{i}$ can still hold and one talks of "form dominance" (Chen and King, 2009). In these models the underlying family symmetry of the Lagrangian $G_{f}$ is completely broken by the combined action of the $\Phi_{i}$ VEVs, and the flavor symmetry of the neutrino mass matrix emerges entirely as an accidental residual symmetry of the quadratic form of Eq. (64) (King, 2010). The symmetry $G_{f}$ plays a less direct role and the name "indirect models" has been used.

\section{EXTENSION TO QUARKS AND GUTS}

Much attention has been devoted to the question whether models with TB mixing in the neutrino sector can be suitably extended to also successfully describe the observed pattern of quark mixings and masses and whether this more complete framework can be made compatible with (supersymmetric) $\mathrm{SU}(5)$ or $\mathrm{SO}(10)$ grand unification. For models with approximate TB mixing in the leptonic sector we first consider the extension to quarks without grand unification and then the more ambitious task of building grand unified models. In GUT models based on $\mathrm{SU}(5) \otimes G_{f}$ or $\mathrm{SO}(10) \otimes G_{f}{ }^{4}$, where $G_{f}$ is a flavor group, all fields in a whole representation of $\mathrm{SU}(5)$ or $\mathrm{SO}(10)$ must have the same transformation properties under $G_{f}$. This poses a strong constraint on the way quarks and leptons have to transform under $G_{f}$.

\section{A. Extension to quarks without GUTs}

The simplest attempts of directly extending models based on $A_{4}$ to quarks have not been satisfactory. The most appealing possibility is to adopt for quarks the same classification scheme under $A_{4}$ that one has used for leptons [see, for example, $\mathrm{Ma}$ and Rajasekaran (2001), Babu et al. (2003), and Altarelli and Feruglio

\footnotetext{
${ }^{4}$ The Pati-Salam group $\mathrm{SU}(4) \otimes \mathrm{SU}(2) \otimes \mathrm{SU}(2)$ has also been considered, for example, by King and Malinsky (2007) and Toorop et al. (2010).
} 
(2006)]. Thus, one tentatively assumes that LH quark doublets $Q$ transform as a triplet 3, while the RH quarks $\left(u^{c}, d^{c}\right),\left(c^{c}, s^{c}\right)$, and $\left(t^{c}, b^{c}\right)$ transform as $1,1^{\prime \prime}$, and $1^{\prime}$, respectively. This leads to $V_{u}=V_{d}$ and to the identity matrix for $V_{\mathrm{CKM}}=V_{u}^{\dagger} V_{d}$ in the lowest approximation. This at first appears as promising: a LO approximation where neutrino mixing is $\mathrm{TB}$ and $V_{\mathrm{CKM}}=1$ is a good starting point. However, there are some problems. First, the corrections to $V_{\mathrm{CKM}}=1$ turn out to be strongly constrained by the leptonic sector because lepton mixing angles are close to the TB values, and, in the simplest models, this constraint leads to a too small $V_{u s}$ (i.e., the Cabibbo angle is rather large in comparison to the allowed shifts from the TB mixing angles) (Altarelli and Feruglio, 2006). Also in these models the quark classification which leads to $V_{\mathrm{CKM}}=1$ is not compatible with $A_{4}$ commuting with $\mathrm{SU}(5)$. An additional consequence of the above assignment is that the top quark mass would arise from a nonrenormalizable dimension-five operator. In that case, to reproduce the top mass we need to compensate the cutoff suppression by some extra dynamical mechanism. Alternatively, we have to introduce a separate symmetry breaking parameter for the quark sector, sufficiently close to the cutoff scale.

Due to this, larger discrete groups have been considered for the description of quarks. A particularly appealing set of models is based on the discrete group $T^{\prime}$, the double covering group of $A_{4}$ (Frampton and Kephart, 1995, 2007; Aranda et al., 2000a, 2000b; Aranda, 2007; Carr and Frampton, 2007; Chen and Mahanthappa, 2007; Feruglio et al., 2007; Ding, 2008; Frampton and Matsuzaki, 2009). As shown in Table II the representations of $T^{\prime}$ are those of $A_{4}$ plus three independent doublets $2,2^{\prime}$, and $2^{\prime \prime}$. The doublets are interesting for the classification of the first two generations of quarks (Barbieri et al., 1996; Pomarol and Tommasini, 1996; Barbieri, Hall, Raby, and Romanino, 1997; Barbieri, Hall, and Romanino, 1997). For example, Feruglio et al. (2007) obtained a viable description; i.e., in the leptonic sector the predictions of the $A_{4}$ model are reproduced, while the $T^{\prime}$ symmetry plays an essential role for reproducing the pattern of quark mixing. However, the classification adopted in this model is again not compatible with grand unification.

\section{B. Extension to quarks within GUTs}

As a result, the group $A_{4}$ was considered by many to be too limited to also describe quarks and to lead to a grand unified description. It has recently been shown (Altarelli et al., 2008) that this negative attitude is not justified and that it is actually possible to construct a viable model based on $A_{4}$ which leads to a grand unified theory (GUT) of quarks and leptons with TB mixing for leptons and with quark (and charged-lepton) masses and mixings compatible with experiment. At the same time this model offers an example of an extra dimensional SU(5) GUT in which a description of all fermion masses and mixings is accomplished. The formulation of $\mathrm{SU}(5)$ in extra dimensions has the usual advantages of avoiding large Higgs representations to break SU(5) and of solving the doublet-triplet splitting problem. The choice of the transformation properties of the two Higgses, $\mathrm{H}_{5}$ and $H_{5}$, has a special role in this model. They are chosen to transform as two different $A_{4}$ singlets, 1 and $1^{\prime}$. As a consequence, mass terms for the Higgs color triplets are not directly allowed and their masses are introduced by orbifolding as described by Kawamura (Witten, 1985; Faraggi, 2001; Kawamura, 2001). In this model, proton decay is dominated by gauge vector boson exchange giving rise to dimension-six operators, while the usual contribution of dimension-five operators is forbidden by the selection rules of the model. Given the large $M_{\mathrm{GUT}}$ scale of SUSY models and the relatively large theoretical uncertainties, the decay rate is within the present experimental limits. A seesaw realization in terms of an $A_{4}$ triplet of RH neutrinos $\nu^{c}$ ensures the correct ratio of light neutrino masses with respect to the GUT scale. In this model extra dimensional effects directly contribute to determine the flavor pattern, in that the two lightest tenplets, $T_{1}$ and $T_{2}$, are in the bulk (with a doubling $T_{i}$ and $T_{i}^{\prime}, i=1,2$, to ensure the correct zero mode spectrum), whereas the pentaplets $F$ and $T_{3}$ are on the brane. The hierarchy of quark and charged-lepton masses and of quark mixings is determined by a combination of extra dimensional suppression factors and $\mathrm{U}(1)_{\mathrm{FN}}$ charges, both of which only apply to the first two generations, while the neutrino mixing angles derive from $A_{4}$ in the usual way. If the extra dimensional suppression factors and the $\mathrm{U}(1)_{\mathrm{FN}}$ charges are switched off, only the third generation masses of quarks and charged-leptons survive. Thus, the charged fermion mass matrices are nearly empty in this limit (not much of $A_{4}$ effects remain) and the quark mixing angles are determined by the small corrections induced by those effects. The model is natural since most of the small parameters in the observed pattern of masses and mixings as well as the necessary vacuum alignment are justified by the symmetries of the model. However, in this case, as in all models based on $\mathrm{U}(1)_{\mathrm{FN}}$, the number of $\mathcal{O}(1)$ parameters is larger than the number of measurable quantities, so that in the quark sector the model can only account for the orders of magnitude (measured in terms of powers of an expansion parameter) and not for the exact values of mass ratios and mixing angles. A moderate fine tuning is only needed to enhance the Cabibbo mixing angle between the first two generations, which would generically be of $\mathcal{O}\left(\lambda_{C}^{2}\right)$.

The problem of constructing GUT models based on $\mathrm{SU}(5) \otimes G_{f}$ or $\mathrm{SO}(10) \otimes G_{f}$ with approximate TB mixing in the leptonic sector has been considered [see, for example, Ma (2005b, 2006b), Ma et al. (2006), Morisi et al. (2007), Altarelli et al. (2008), Bazzocchi, Frigerio, and Morisi (2008), Grimus and Kuhbock (2008), Bazzocchi, Morisi, Picariello, and Torrente-Lujan (2009), Ciafaloni et al. (2009), and Antusch et al. (2010) based on $A_{4}$ ]. In our opinion most of the models are incomplete (for example, the crucial issue of VEV alignment is not really 
treated in depth as it should) and/or involve a number of unjustified ad hoc fine tuning of parameters. An interesting model based on $\mathrm{SU}(5) \otimes T^{\prime}$ is discussed by Chen and Mahanthappa (2007). In this model the SU(5) tenplets $T_{3}$ and $T_{a}(a=1,2)$ of the third and first two generations are classified as 1 and 2 of $T^{\prime}$, respectively, while the SU(5) pentaplets are in a 3 of $T^{\prime}$. This model provides a good description of fermion masses and mixings and appears simpler than the model of Altarelli et al. (2008), which is also based on SU(5). However, the model of Chen and Mahanthappa (2007) is fine tuned. In fact, one does not understand how it is possible that, for example, the electron and muon masses can come out so widely different as observed, given that in this model their left and right components separately transform in an identical way under $T^{\prime}$. The reason is that in the second term of Eq. (5) of Chen and Mahanthappa (2007), only one of three possible contractions has been taken into account. If the missing ones, which are also allowed by the assumed symmetry properties, are included with generic coefficients, one in fact finds that the $e$ and $\mu$ masses are of the same order in the absence of fine tuning. Given that the expansion parameter in the model is of $\mathcal{O}\left(\lambda_{C}\right)$ the fine tuning which is needed is large. One possible way out would be to invoke some ultraviolet completion of the model where particular heavy field exchanges could justify the presence of only the desired couplings after the heavy fields are integrated out. Also, in the model of Chen and Mahanthappa (2007) there is no discussion of the origin of the required vacuum alignment. Recently some GUT models based on SU(5) $\times S_{4}$ have appeared (Hagedorn et al., 2010; Ishimori et al., 2010). In these models the first two generation fermions are in also the same $S_{4}$ representations (either a doublet, for tenplets, or a triplet, for pentaplets). In the absence of an additional principle the electron and muon masses should naturally be of the same order. Ishimori et al. (2010) obtained the vanishing of the electron mass at LO by the ad hoc choice of one particular minimum of the scalar potential among a continuous family of degenerate solutions [see their Eqs. (70) and (71)]. In the case of Hagedorn et al. (2010) the problem is solved by introducing new heavy particles with suitable interactions that, once integrated out, produce the desired structure for the mass matrix.

As for the models based on $\mathrm{SO}(10) \otimes G_{f}$ we select two recent examples with $G_{f}=S_{4}$ (Dutta et al., 2009, 2010) and $G_{f}=P S L_{2}(7)$ (King and Luhn, 2009b). The case of $\mathrm{SO}(10)$ is even more difficult than that of $\mathrm{SU}(5)$ because the neutrino sector is tightly related to that of quarks and charged leptons as all belong to the 16 of $\mathrm{SO}(10)$ [for a general analysis of $\mathrm{SO}(10) \otimes A_{4}$, see Bazzocchi, Frigerio, and Morisi (2008)]. The strategy adopted by Dutta et al. (2009, 2010) and King and Luhn (2009b), as well as in other $\mathrm{SO}(10)$ models, is as follows. One considers renormalizable fermion mass terms with the Higgs multiplets of the $\mathrm{SO}(10) 10$ ( $h$ terms) and 126 ( $f$ terms) representations. The Majorana neutrino mass matrix arises from the 126 . One assumes that the dominant con- tribution to the Dirac masses of fermions is from the $h$ terms with small corrections from the $f$ terms. In first approximation the $h$ contribution is a matrix of rank 1 with only the third generation mass being nonvanishing. The light fermion masses and the quark mixings then arise from the $f$ terms (and from some possible extra terms). The third family dominance is obtained by a term with a double flavon factor of Dutta et al. (2009, 2010) (based on $S_{4}$ ) which then makes it particularly difficult to keep the corrective $f$ terms small (for this fine tuning is needed or a suitable ultraviolet completion). King and Luhn (2009b) induced the dominant $h$ terms by a single $\mathrm{PSL}_{2}(7)$ sextet flavon [the existence of complex three- and of six-dimensional representations is the peculiarity of $\left.\mathrm{PSL}_{2}(7)\right]$. In both models in the neutrino sector one has a sum of types I and II seesaw contributions of the form

$$
m_{\nu}=f v_{L}-m_{D}^{T} \frac{1}{f v_{R}} m_{D},
$$

where the first term is from the exchange of a triplet Higgs with VEV proportional to $v_{L}$ while the second term is from type I seesaw with $\mathrm{RH}$ mass proportional to $v_{R}$. One must assume that the first term is dominant and the second is negligible. Then the leading approximation for the fermion Dirac masses is from the $h$ terms and for neutrino masses from the $f$ terms. The $f$ terms are diagonalized by the TB mixing unitary matrix. In this way the connection between quarks and neutrinos is relaxed and a completely different pattern of mixing can be realized in the two sectors. Clearly for the $f v_{L}$ dominance in Eq. (65) one needs $v_{L} \gg v^{2} / v_{R}$ with $v \sim h / f$. This needs widely different scales for $v_{L}$ and $v_{R}$ in the model and much of the description of the corresponding dynamics, along the lines of Goh et al. (2004) and Mohapatra et al. (2007), remains to be studied in detail. In both of these models the discussion of the alignment is not satisfactory. In particular King and Luhn (2009b) only proved that the arbitrary coefficients appearing in the most general allowed superpotential can be fitted to lead to the required ratios of components in the VEVs (while for a natural model one would require that the alignment automatically follows in a whole region of the parameter space). In conclusion, the problem of constructing a satisfactory natural model based on $\mathrm{SO}(10)$ with built-in TB mixing at the $\mathrm{LO}$ approximation remains open.

\section{THE $S_{4}$ GROUP AND BM MIXING}

If one takes the alternative view that the agreement with TB mixing is accidental and is rather oriented to consider weak complementarity as a guiding principle, then a better starting point could be BM mixing. In the BM scheme $\tan ^{2} \theta_{12}=1$, to be compared with the latest experimental determination: $\tan ^{2} \theta_{12}=0.45 \pm 0.04$ (at $1 \sigma$ ) (Strumia and Vissani, 2006; Bandyopadhyay et al., 2008; Fogli et al., 2008a, 2008b; Gonzalez-Garcia and Maltoni, 2008b; Maltoni and Schwetz, 2008; Schwetz et al., 2008) 
TABLE VI. Transformation properties of all the fields.

\begin{tabular}{lccccccccccccccc}
\hline \hline & $l$ & $e^{c}$ & $\mu^{c}$ & $\tau^{c}$ & $\nu^{c}$ & $h_{u, d}$ & $\theta$ & $\phi_{l}$ & $\chi_{l}$ & $\psi_{l}^{0}$ & $\chi_{l}^{0}$ & $\xi_{\nu}$ & $\phi_{\nu}$ & $\xi_{\nu}^{0}$ & $\phi_{\nu}^{0}$ \\
\hline$S_{4}$ & 3 & 1 & $1^{\prime}$ & 1 & 3 & 1 & 1 & 3 & $3^{\prime}$ & 2 & $3^{\prime}$ & 1 & 3 & 1 & 3 \\
$Z_{4}$ & 1 & -1 & $-i$ & $-i$ & 1 & 1 & 1 & $i$ & $i$ & -1 & -1 & 1 & 1 & 1 & 1 \\
$\mathrm{U}(1)_{\mathrm{FN}}$ & 0 & 2 & 1 & 0 & 0 & 0 & -1 & 0 & 0 & 0 & 0 & 0 & 0 & 0 & 0 \\
$\mathrm{U}(1)_{R}$ & 1 & 1 & 1 & 1 & 1 & 1 & 0 & 0 & 0 & 2 & 2 & 0 & 0 & 2 & 2 \\
\hline \hline
\end{tabular}

(see Fig. 2), so that a rather large nonleading correction is needed, as mentioned. A discrete group approach can also work for BM mixing. We now summarize a model (Altarelli et al., 2009) based on $S_{4}$ that leads to BM mixing in first approximation while the agreement with the data is restored by large NLO corrections that arise from the charged-lepton sector.

The group $S_{4}$ is particularly suitable for reproducing BM mixing in LO because the unitary matrices $S_{\mathrm{BM}}$, given in Eq. (22), and $T_{\mathrm{BM}}$, given in Eq. (25), directly provide a presentation of $S_{4}$. We recall that $S_{\mathrm{BM}}$ leaves invariant the most general mass matrix for BM mixing in the charged-lepton diagonal basis [Eq. (20)], while $T_{\mathrm{BM}}$ leaves invariant the most general diagonal matrix $m_{e}^{\dagger} m_{e}$ for charged leptons [see Eqs. (21) and (23)]. In fact, from Table II, we see that a possible presentation of $S_{4}$ is given by

$$
A^{4}=B^{2}=(A B)^{3}=1 \text {. }
$$

In terms of $3 \times 3$ matrices, we can make the identifications $A=T_{\mathrm{BM}}$ and $B=S_{\mathrm{BM}}$, and Eq. (66) are satisfied. As was the case for the $A_{4}$ models, in this model the invariance under $A_{23}$, which is also necessary to specify BM mixing according to Eq. (21), arises again accidentally as a consequence of the specific field content and is limited to the contribution of the dominant terms to the neutrino mass matrix.

In the model the three generations of LH lepton doublets $l$ and RH neutrinos $\nu^{c}$ transform as two triplets 3, while the RH charged leptons $e^{c}, \mu^{c}$, and $\tau^{c}$ transform as $1,1^{\prime}$, and 1 , respectively. The $S_{4}$ symmetry is then broken by suitable triplet flavons. All the flavon fields are singlets under the standard model gauge group. Additional symmetries are needed, as usual, to prevent unwanted couplings and to obtain a natural hierarchy among $m_{e}, m_{\mu}$, and $m_{\tau}$. The complete flavor symmetry of the model is $S_{4} \times Z_{4} \times \mathrm{U}(1)_{\mathrm{FN}}$. A flavon $\theta$, carrying a negative unit of the $\mathrm{U}(1)_{\mathrm{FN}}$ charge $F$, acquires a vacuum expectation value $(\mathrm{VEV})$ and breaks $\mathrm{U}(1)_{\mathrm{FN}}$. A supersymmetric context is adopted, so that two Higgs doublets $h_{u, d}$, invariant under $S_{4}$, are present in the model as well as the $\mathrm{U}(1)_{R}$ symmetry related to $R$ parity and the driving fields in the flavon superpotential. Supersymmetry also helps producing and maintaining the hierarchy $\left\langle h_{u, d}\right\rangle=v_{u, d} \ll \Lambda$ where $\Lambda$ is the cutoff scale of the theory.

The fields in the model and their classification under the symmetry are summarized in Table VI. The fields $\psi_{l}^{0}$, $\chi_{l}^{0}, \xi_{\nu}^{0}$, and $\phi_{\nu}^{0}$ are the driving fields.
The complete superpotential can be written as $w=w_{l}$ $+w_{\nu}+w_{d}$. The $w_{d}$ term is responsible for the alignment. It was discussed by Altarelli et al. (2009) and this discussion will not be repeated here. The terms $w_{l}$ and $w_{\nu}$ determine the lepton mass matrices [we indicate with $(\cdots)$ the singlet 1 , with $(\cdots)^{\prime}$ the singlet $1^{\prime}$, and with $(\cdots)_{V}\left(V=2,3,3^{\prime}\right)$ the representation $\left.V\right]$,

$$
\begin{aligned}
w_{l}= & \frac{y_{e}^{(1)}}{\Lambda^{2}} \frac{\theta^{2}}{\Lambda^{2}} e^{c}\left(l \phi_{l} \phi_{l}\right)+\frac{y_{e}^{(2)}}{\Lambda^{2}} \frac{\theta^{2}}{\Lambda^{2}} e^{c}\left(l \chi_{l} \chi_{l}\right) \\
& +\frac{y_{e}^{(3)}}{\Lambda^{2}} \frac{\theta^{2}}{\Lambda^{2}} e^{c}\left(l \phi_{l} \chi_{l}\right)+\frac{y_{\mu}}{\Lambda} \frac{\theta}{\Lambda} \mu^{c}\left(l \chi_{l}\right)^{\prime}+\frac{y_{\tau}}{\Lambda} \tau^{c}\left(l \phi_{l}\right) \\
& +\cdots \\
w_{\nu}= & y\left(\nu^{c} l\right)+M \Lambda\left(\nu^{c} \nu^{c}\right)+a\left(\nu^{c} \nu^{c} \xi_{\nu}\right)+b\left(\nu^{c} \nu^{c} \phi_{\nu}\right)+\cdots
\end{aligned}
$$

where $a$ and $b$ are complex coefficients. To keep our formulas compact, we omit to write the Higgs fields $h_{u, d}$. For instance, $y_{\tau} \tau^{\mathcal{c}}\left(l \phi_{l}\right) / \Lambda$ stands for $y_{\tau} \mathcal{\tau}\left(l \phi_{l}\right) h_{d} / \Lambda$ and $y\left(\nu^{c} l\right)$ stands for $y\left(\nu^{c} l\right) h_{u}$. The powers of the cutoff $\Lambda$ also take into account the presence of the omitted Higgs fields. Note that the parameters $M, M_{\phi}, M_{\xi}$, and $M_{\xi}^{\prime}$ defined above are dimensionless. In the above expression for the superpotential $w$, only the lowest order operators in an expansion in powers of $1 / \Lambda$ are explicitly shown. Dots stand for higher-dimensional operators that will be discussed later. The stated symmetries ensure that, for the leading terms, the flavons that appear in $w_{l}$ cannot contribute to $w_{\nu}$ and vice versa.

The potential corresponding to $w_{d}$ possesses an isolated minimum for the following VEV configuration:

$$
\begin{aligned}
& \frac{\left\langle\phi_{l}\right\rangle}{\Lambda}=\left(\begin{array}{l}
0 \\
1 \\
0
\end{array}\right) A, \quad \frac{\left\langle\chi_{l}\right\rangle}{\Lambda}=\left(\begin{array}{l}
0 \\
0 \\
1
\end{array}\right) B, \\
& \frac{\left\langle\phi_{\nu}\right\rangle}{\Lambda}=\left(\begin{array}{c}
0 \\
1 \\
-1
\end{array}\right) C, \quad \frac{\left\langle\xi_{\nu}\right\rangle}{\Lambda}=D,
\end{aligned}
$$

where the factors $A, B, C$, and $D$ should obey to the relations

$$
\sqrt{3} f_{1} A^{2}+\sqrt{3} f_{2} B^{2}+f_{3} A B=0
$$




$$
D=-\frac{M_{\phi}}{g_{2}}, \quad C^{2}=\frac{g_{2}^{2} M_{\xi}^{2}+g_{3} M_{\phi}^{2}-g_{2} M_{\phi} M_{\xi}^{\prime}}{2 g_{2}^{2} g_{4}} .
$$

Similarly, the Froggatt-Nielsen flavon $\theta$ gets a VEV, determined by the $D$ term associated to the local $\mathrm{U}(1)_{\mathrm{FN}}$ symmetry, and it is denoted by

$$
\frac{\langle\theta\rangle}{\Lambda}=t
$$

With this VEV configuration, the charged-lepton mass matrix is diagonal,

$$
m_{l}=\left(\begin{array}{ccc}
\left(y_{e}^{(1)} B^{2}-y_{e}^{(2)} A^{2}+y_{e}^{(3)} A B\right) t^{2} & 0 & 0 \\
0 & y_{\mu} B t & 0 \\
0 & 0 & y_{\tau} A
\end{array}\right) v_{d},
$$

so that at LO there is no contribution to the $U_{\mathrm{PMNS}}$ mixing matrix from the diagonalization of charged-lepton masses. In the neutrino sector for the Dirac and $\mathrm{RH}$ Majorana matrices we have

$$
\begin{aligned}
& m_{\nu}^{D}=\left(\begin{array}{lll}
1 & 0 & 0 \\
0 & 0 & 1 \\
0 & 1 & 0
\end{array}\right) y v_{u}, \\
& M_{N}=\left(\begin{array}{ccc}
2 M+2 a D & -2 b C & -2 b C \\
-2 b C & 0 & 2 M+2 a D \\
-2 b C & 2 M+2 a D & 0
\end{array}\right) \Lambda .
\end{aligned}
$$

The matrix $M_{N}$ can be diagonalized by the BM mixing matrix $U_{\mathrm{BM}}$, which represents the full lepton mixing at the LO, and the eigenvalues are

$$
\begin{aligned}
& M_{1}=2|M+a D-\sqrt{2} b C| \Lambda, \\
& M_{2}=2|M+a D+\sqrt{2} b C| \Lambda, \quad M_{3}=2|M+a D| \Lambda .
\end{aligned}
$$

After the seesaw mechanism, since the Dirac neutrino mass matrix commutes with $M_{N}$ and its square is a matrix proportional to unity, the light neutrino Majorana mass matrix, given by the seesaw relation $m_{\nu}$ $=\left(m_{\nu}^{D}\right)^{T} M_{N}^{-1} m_{\nu}^{D}$, is also diagonalized by the BM mixing matrix and the eigenvalues are

$$
\begin{aligned}
& \left|m_{1}\right|=\frac{\left|y^{2}\right| v_{u}^{2}}{2|M+a D-\sqrt{2} b C|} \frac{1}{\Lambda}, \\
& \left|m_{2}\right|=\frac{\left|y^{2}\right| v_{u}^{2}}{2|M+a D+\sqrt{2} b C|} \frac{1}{\Lambda}, \quad\left|m_{3}\right|=\frac{\left|y^{2}\right| v_{u}^{2}}{2|M+a D|} \frac{1}{\Lambda} .
\end{aligned}
$$

The light neutrino mass matrix depends on only two effective parameters; at LO the terms $M$ and $a D$ enter the mass matrix in the combination $F \equiv M+a D$. The coefficients $y_{e}^{(i)}, y_{\mu}, y_{\tau}, y, a$, and $b$ are all expected to be of $\mathcal{O}(1)$. A priori $M$ could be of $\mathcal{O}(1)$, corresponding to a $\mathrm{RH}$ neutrino Majorana mass of $\mathcal{O}(\Lambda)$, but, actually, it must be of the same order as $C$ and $D$. In the context of a grand unified theory this would correspond to the re- quirement that $M$ is of $\mathcal{O}\left(M_{\mathrm{GUT}}\right)$ rather than of $\mathcal{O}\left(M_{\text {Planck }}\right)$.

We expect a common order of magnitude for the VEVs (scaled by the cutoff $\Lambda$ ),

$$
A \sim B \sim v, \quad C \sim D \sim v^{\prime} .
$$

However, due to the different minimization conditions that determine $(A, B)$ and $(C, D)$, we may tolerate a moderate hierarchy between $v$ and $v^{\prime}$. Similarly the order of magnitude of $t$ is, in principle, unrelated to those of $v$ and $v^{\prime}$. It is possible to estimate the values of $v$ and $t$ by looking at the mass ratios of charged leptons (while $v^{\prime}$ only enters in the neutrino sector), and the result is that $t \sim 0.06$ and $v \sim 0.08$ [modulo coefficients of $\mathcal{O}(1)$ ].

So far we have shown that, at LO, we have diagonal and hierarchical charged leptons together with the exact $\mathrm{BM}$ mixing for neutrinos. It is clear that substantial NLO corrections are needed to bring the model to agree with the data on $\theta_{12}$. A crucial feature of the model is that the neutrino sector flavons $\phi_{\nu}$ and $\xi_{\nu}$ are invariant under $Z_{4}$ which is not the case for the charged-lepton sector flavons $\phi_{l}$ and $\chi_{l}$. The consequence is that $\phi_{\nu}$ and $\xi_{\nu}$ can contribute at NLO to the corrections in the charged-lepton sector, while at NLO $\phi_{l}$ and $\chi_{l}$ cannot modify the neutrino sector couplings. As a result the dominant corrections to the BM mixing matrix only occur at NLO through the diagonalization of the charged leptons. In fact, at NLO the neutrino mass matrix is still diagonalized by $U_{\mathrm{BM}}$ but the mass matrix of charged leptons is no more diagonal. Including these additional terms from the diagonalization of charged leptons the $U_{\text {PMNS }}$ matrix can be written as

$$
U_{\mathrm{PMNS}}=U_{l}^{\dagger} U_{\mathrm{BM}},
$$

and therefore the corrections from $U_{l}$ affect the neutrino mixing angles at NLO according to

$$
\begin{aligned}
& \sin ^{2} \theta_{12}=\frac{1}{2}-\frac{1}{\sqrt{2}}\left(V_{12}+V_{13}\right) v^{\prime}, \\
& \sin ^{2} \theta_{23}=\frac{1}{2}, \\
& \sin \theta_{13}=\frac{1}{\sqrt{2}}\left(V_{12}-V_{13}\right) v^{\prime},
\end{aligned}
$$

where the coefficients $V_{i j}$ arise from $U_{l}$. By comparing these expressions with the current experimental values of the mixing angles in Table I, we see that, to correctly reproduce $\theta_{12}$, we need a parameter $v^{\prime}$ of the order of the Cabibbo angle $\lambda_{C}$. Moreover, barring cancellations of or among some of the $V_{i j}$ coefficients, $\theta_{13}$ is also corrected by a similar amount, while $\theta_{23}$ is unaffected at the NLO. A salient feature of this model is that, at NLO accuracy, the large corrections of $\mathcal{O}\left(\lambda_{C}\right)$ only apply to $\theta_{12}$ and $\theta_{13}$ while $\theta_{23}$ is unchanged at this order. As a correction of $\mathcal{O}\left(\lambda_{C}\right)$ to $\theta_{23}$ is hardly compatible with the present data (see Table I) this feature is crucial for the phenomenological success of this model. It is easy to see 
that this essential property depends on the selection in the neutrino sector of flavons $\xi_{\nu}$ and $\phi_{\nu}$ that transform as 1 and 3 of $S_{4}$, respectively. If, for example, the singlet $\xi_{\nu}$ is replaced by a doublet $\psi_{\nu}$ (and correspondingly the singlet driving field $\xi_{\nu}^{0}$ is replaced by a doublet $\psi_{\nu}^{0}$ ), all other quantum numbers being the same, one can construct a variant of the model along similar lines, but in this case all the three mixing angles are corrected by terms of the same order. This confirms that a particular set of $S_{4}$ breaking flavons is needed in order to preserve $\theta_{23}$ from taking as large corrections as the other two mixing angles.

This discussion applies at the NLO and we expect that at the next-to-next-to-leading-order the value of $\theta_{23}$ will eventually be modified with deviations of about $\mathcal{O}\left(\lambda_{C}^{2}\right)$. The next generation of experiments, in particular those exploiting a high intensity neutrino beam, will probably reduce the experimental error on $\theta_{23}$ and the sensitivity on $\theta_{13}$ to few degrees. All quantitative estimates are clearly affected by large uncertainties due to the presence of unknown parameters of order 1 , but in this model a value of $\theta_{13}$ much smaller than the present upper bound would be unnatural. If in the forthcoming generation of experiments no significant deviations from zero of $\theta_{13}$ will be detected, this construction will be strongly disfavored.

\section{LEPTON FLAVOR VIOLATION}

Neutrino oscillations provide evidence of flavor conversion in the lepton sector. This indicates that lepton flavor violation (LFV) might take place, at least at some level, also in other processes such as those involving charged leptons. Flavor violating decays of charged leptons, strictly forbidden in the SM, are indeed allowed as soon as neutrino mass terms are considered. If neutrino masses are the only source of LFV, the effects are too small to be detected, but in most extensions of the SM where new particles and new interactions with a characteristic scale $\Lambda_{N P}$ are included, the presence of new sources of flavor violation, in both quark and lepton sectors, is a generic feature. The scale $\Lambda_{N P}$ can be much smaller than the cutoff scale $\Lambda$ introduced before. Indeed, there are several indications suggesting new physics at the $\mathrm{TeV}$ scale, such as a successful gauge coupling unification, viable solutions to the hierarchy problem, and realistic dark matter candidates. In a low-energy description, the associated effects can be parametrized by higher-dimensional operators. The dominant terms are represented by dimension-six operators suppressed by two powers of $\Lambda_{N P}$,

$$
\begin{aligned}
\mathcal{L}_{\text {eff }}= & i \frac{e}{\Lambda_{N P}^{2}} e_{i}^{c} H^{\dagger} \sigma^{\mu \nu} F_{\mu \nu} \mathcal{Z}_{i j} l_{j}+\frac{1}{\Lambda_{N P}^{2}}[\text { four } \\
& \text {-fermion operators }]+ \text { H.c. },
\end{aligned}
$$

where $e$ is the electric charge and $\mathcal{Z}_{i j}$ denotes an adimensional complex matrix with indices in flavor space. If the underlying theory is weakly interacting with a typical coupling constant $g_{N P}$ and predicts new particles of mass $m_{N P}$, we expect $\Lambda_{N P} \approx 4 \pi m_{N P} / g_{N P}$. The present bounds on the branching ratios (Raidal et al., 2008) of the rare charged-lepton decays set stringent limits on combinations of the scale $\Lambda_{N P}$ and the coefficients of the involved operators. For instance, from $B(\mu \rightarrow e \gamma)<1.2$ $\times 10^{-11}$ (Brooks et al., 1999; Adam et al., 2010) we get $\left|\mathcal{Z}_{\mu e}\right|<10^{-8} \times\left[\Lambda_{N P}(\mathrm{TeV}) / 1 \mathrm{TeV}\right]^{2}$. Typically, for coefficients of order 1 , the existing bounds require a large scale $\Lambda_{N P}$, several orders of magnitude larger than the $\mathrm{TeV}$ scale. Conversely, to allow for new physics close to the $\mathrm{TeV}$ scale coefficients much smaller than 1 are required, which may indicate the effect of a flavor symmetry.

In theories with a flavor symmetry group $G_{f}$ spontaneously broken by a set of small parameters $\varepsilon$, the coefficients of the effective Lagrangian in Eq. (81) become functions of $\varepsilon$. The low-energy Lagrangian of Eq. (81) is derived from the theory defined close to the cutoff scale $\Lambda$, where all operators are invariant under $G_{f}$, thanks to their dependence on the flavon multiplets. Below the flavor symmetry breaking scale the flavons are replaced by their VEVs, which enter the coefficients of $\mathcal{L}_{\text {eff }}$ through the dimensionless combination $\varepsilon \approx \mathrm{VEV} / \Lambda$. Exploiting the smallness of the parameters $\varepsilon$ we can keep in $\mathcal{L}_{\text {eff }}$ the first few terms of a power series expansion. For instance,

$$
\mathcal{Z}_{i j} \equiv \mathcal{Z}_{i j}(\varepsilon)=\mathcal{Z}_{i j}^{(0)}+\mathcal{Z}_{i j}^{(1)} \varepsilon+\mathcal{Z}_{i j}^{(2)} \varepsilon^{2}+\cdots
$$

Note that the same symmetry breaking parameters that control lepton masses and mixing angles also control the flavor pattern of the operators in $\mathcal{L}_{\text {eff. }}$ This result is interesting in several respects. First, the presence of the factors $\varepsilon^{n}$ can help in suppressing the rates of rare charged-lepton decays while allowing for a relatively small and accessible scale $\Lambda_{N P}$. Second, once the above expansion has been determined in a given model, it could be possible to establish characteristic relations among LFV processes as a consequence of flavor symmetries and their pattern of symmetry breaking. Finally, if $\Lambda_{N P}$ is sufficiently small, this opens the possibility that new particles might be produced and detected at the CERN Large Hadron Collider (LHC), with features that could additionally confirm or reject the assumed symmetry pattern. This allows, at least in principle, to realize an independent test of the flavor symmetry in the chargedlepton sector. While the size of the scale $\Lambda_{N P}$ could be relatively small, in our presentation we assume that the flavor scale or cutoff $\Lambda$ is extremely large, possibly as large as the GUT scale. Then all low-energy effects due to the flavon dynamics are essentially those associated to their VEVs, which enter the effective higherdimensional operators through the dimensionless combination $\epsilon$. Virtual flavon exchanges give rise to other higher-dimensional operators which are depleted by inverse power of $\Lambda$ and can be safely neglected. A much richer variety of effects due to the flavor dynamics would be possible if the scale $\Lambda$ was much smaller, close 
to the $100 \mathrm{TeV}$ energy range, but we do not consider this possibility here.

The effects described by $\mathcal{L}_{\text {eff }}$ are well known. In a field basis where the kinetic terms are canonical and the charged-lepton mass matrix is diagonal the real and imaginary parts of the diagonal matrix elements $\mathcal{Z}_{i i}$ are proportional to the anomalous magnetic dipole moments (MDMs) $a_{i}$ and to the electric dipole moments (EDMs) $d_{i}$ of charged leptons, respectively,

$$
a_{i}=2 m_{i}^{c h} \frac{v}{\sqrt{2} \Lambda_{N P}^{2}} \operatorname{Re} \mathcal{Z}_{i i}, \quad d_{i}=e \frac{v}{\sqrt{2} \Lambda_{N P}^{2}} \operatorname{Im} \mathcal{Z}_{i i}
$$

The off-diagonal elements $\mathcal{Z}_{i j}$ describe the amplitudes for the radiative decays of the charged leptons,

$$
R_{i j}=\frac{B\left(l_{i} \rightarrow l_{j} \gamma\right)}{B\left(l_{i} \rightarrow l_{j} \nu_{i} \bar{\nu}_{j}\right)}=\frac{12 \sqrt{2} \pi^{3} \alpha}{G_{F}^{3} m_{i}^{c h^{2}} \Lambda_{N P}^{4}}\left(\left|\mathcal{Z}_{i j}\right|^{2}+\left|\mathcal{Z}_{j i}\right|^{2}\right),
$$

where $\alpha$ is the fine structure constant, $G_{F}$ is the Fermi constant, and $m_{i}^{c h}$ is the mass of the lepton $l_{i}$. Finally, the four-fermion operators, together with the dipole operators controlled by $\mathcal{Z}$, describe other flavor violating processes such as $\mu \rightarrow e e e, \tau \rightarrow \mu \mu \mu$, and $\tau \rightarrow e e e$.

An interesting example of flavor symmetry is that of minimal flavor violation (MFV) (Sekhar Chivukula and Georgi, 1987; Hall and Randall, 1990; Ciuchini et al., 1998; Buras et al., 2001; D'Ambrosio et al., 2002; Cirigliano et al., 2005; Cirigliano and Grinstein, 2006; Davidson and Palorini, 2006; Grinstein et al., 2007) whose (minimal) flavor symmetry group in the lepton sector is $G_{f}=\mathrm{SU}(3)_{e^{c}} \times \mathrm{SU}(3)_{l}$. Electroweak singlets $e^{c}$ and doublets $l$ transform as $(3,1)$ and $(1, \overline{3})$, respectively. The flavon fields or better their VEVs are the Yukawa couplings of the charged leptons $Y_{l}=m_{l} / v$ and the adimensional coupling constants $\eta$ of the five-dimensional operator $\mathrm{O}_{5}$ in Eq. (2). They transform as $(\overline{3}, 3)$ and $(1,6)$, respectively. On a basis where the charged leptons are diagonal, we have

$$
Y_{l}=\frac{\sqrt{2}}{v} m_{l}^{\text {diag }}, \quad \eta=\frac{M}{v^{2}} U^{*} m_{\nu}^{\text {diag }} U^{\dagger},
$$

where $M$ denotes the mass scale suppressing the operator $\mathrm{O}_{5}$. In MFV models the leading off-diagonal elements of $\mathcal{Z}_{i j}$ are given by

$$
\begin{aligned}
\mathcal{Z}_{i j} & =c\left(Y_{l} \eta^{\dagger} \eta\right)_{i j} \\
& =\sqrt{2} c \frac{m_{i}^{c h}}{v} \frac{M^{2}}{v^{4}}\left[\Delta m_{\mathrm{sol}}^{2} U_{i 2} U_{j 2}^{*} \pm \Delta m_{\mathrm{atm}}^{2} U_{i 3} U_{j 3}^{*}\right],
\end{aligned}
$$

where $c$ is an overall coefficient of order 1 and the plus (minus) sign refers to the case of normal (inverted) hierarchy. We see that, due to the presence of the ratio $M^{2} / v^{2}$, the overall scale of these matrix elements is poorly constrained. This is due to the fact that MFV does not restrict the overall strength of the coupling constants $\eta$, apart from the requirement that they remain in the perturbative regime. Very small or relatively large (but smaller than 1) $\eta$ can be accommodated by adjust- ing the scale $M$. Thus, even after fixing $\Lambda_{N P}$ close to the $\mathrm{TeV}$ scale, in MFV the nonobservation of $l_{i} \rightarrow l_{j} \gamma$ could be justified by choosing a small $M$, while a positive signal in $\mu \rightarrow e \gamma$ with a branching ratio in the range of 1.2 $\times 10^{-11}-1.2 \times 10^{-13}$ could also be fitted by an appropriate $M$, apart from a small region of the $\theta_{13}$ angle, around $\theta_{13} \approx 0.02$ where a cancellation can take place in the lefthand side of Eq. (86). The dependence on the scales $M$ and $\Lambda_{N P}$ can be eliminated by considering ratios of branching ratios. For instance,

$$
\frac{R_{\mu e}}{R_{\tau \mu}}=\left|\frac{2 \Delta m_{\mathrm{sol}}^{2}}{3 \Delta m_{\mathrm{atm}}^{2}} \pm \sqrt{2} \sin \theta_{13} e^{i \delta}\right|^{2}<1,
$$

where we took the TB ansatz to fix $\theta_{12}$ and $\theta_{23}$. We see that $B(\mu \rightarrow e \gamma)<B(\tau \rightarrow \mu \gamma)$ always in MFV. Moreover, for $\theta_{13}$ above approximately $0.07, \quad B(\mu \rightarrow e \gamma)<1.2$ $\times 10^{-11}$ implies $B(\tau \rightarrow \mu \gamma)<10^{-9}$. For $\theta_{13}$ below 0.07, apart possibly from a small region around $\theta_{13} \approx 0.02$, both the transitions $\mu \rightarrow e \gamma$ and $\tau \rightarrow \mu \gamma$ might be above the sensitivity of the future experiments. The present limits are $B(\tau \rightarrow \mu \gamma)<1.6 \times 10^{-8}$ and $B(\tau \rightarrow e \gamma)<9.4$ $\times 10^{-8}$. A future super-B factory might improve them by about one order of magnitude. In the SUSY case there are two doublets in the low-energy Lagrangian and we should take into account the $\tan \beta$ dependence.

A different result for the matrix $\mathcal{Z}$ is obtained in the model described in Sec. IV where $G_{f}=A_{4} \times Z_{3} \times \mathrm{U}(1)_{\mathrm{FN}}$. Starting from the relevant set of invariant operators, after the breaking of the flavor and electroweak symmetries and after moving to a basis with canonical kinetic terms and diagonal mass matrix for charged leptons, we find (Feruglio et al., 2008, 2009b)

$$
\mathcal{Z}=\left(\begin{array}{ccc}
\mathcal{O}\left(t^{2} \varepsilon\right) & \mathcal{O}\left(t^{2} \varepsilon^{2}\right) & \mathcal{O}\left(t^{2} \varepsilon^{2}\right) \\
\mathcal{O}\left(t \varepsilon^{2}\right) & \mathcal{O}(t \varepsilon) & \mathcal{O}\left(t \varepsilon^{2}\right) \\
\mathcal{O}\left(\varepsilon^{2}\right) & \mathcal{O}\left(\varepsilon^{2}\right) & \mathcal{O}(\varepsilon)
\end{array}\right)
$$

where each matrix element is known only up to an unknown order-1 dimensionless coefficient. There are two independent symmetry breaking parameters. The parameter $t=\langle\theta\rangle / \Lambda$ controls the charged-lepton mass hierarchy and $\varepsilon=v_{T} / \Lambda$ describes the breaking of $A_{4}$. Note that the uncertainty in the overall scale of the matrix elements $\mathcal{Z}_{i j}$ is related to the parameter $\varepsilon$ and is much smaller than the corresponding uncertainty in MFV. We can see that MDMs and EDMs arise at the first order in the parameter $\varepsilon$. By assuming that the unknown coefficients have absolute values and phases of order 1, from Eqs. (83) and (88) we have

$$
a_{i}=\mathcal{O}\left(2 m_{i}^{c h^{2}} / \Lambda_{N P}^{2}\right), \quad d_{i}=\mathcal{O}\left(e m_{i}^{c h} / \Lambda_{N P}^{2}\right) .
$$

From the existing limits on MDMs and EDMs and using Eqs. (89) as exact equalities we find the results shown in Table VII.

Concerning the flavor violating dipole transitions, from Eq. (88) we see that the dominant contribution to the rate for $l_{i} \rightarrow l_{j} \gamma$ is given by 
TABLE VII. Experimental limits on lepton MDMs and EDMs and corresponding bounds on the scale $\Lambda_{N P}$ derived from Eq. (89). The data on the $\tau$ lepton have not been reported since they are much less constraining. For the anomalous magnetic moment of the muon, $\delta a_{\mu}$ stands for the deviation of the experimental central value from the SM expectation (Bennett et al., 2004; Passera et al., 2009).

\begin{tabular}{lc}
\hline \hline$d_{e}<1.6 \times 10^{-27} e \mathrm{~cm}$ & $\Lambda_{N P}>80 \mathrm{TeV}$ \\
$d_{\mu}<2.8 \times 10^{-19} e \mathrm{~cm}$ & $\Lambda_{N P}>80 \mathrm{GeV}$ \\
$\delta a_{e}<3.8 \times 10^{-12}$ & $\Lambda_{N P}>350 \mathrm{GeV}$ \\
$\delta a_{\mu}=302 \pm 88 \times 10^{-11}$ & $\Lambda_{N P} \approx 2.7 \mathrm{TeV}$ \\
\hline \hline
\end{tabular}

$$
\frac{B\left(l_{i} \rightarrow l_{j} \gamma\right)}{B\left(l_{i} \rightarrow l_{j} \nu_{i} \bar{\nu}_{j}\right)}=\frac{48 \pi^{3} \alpha}{G_{F}^{2} \Lambda_{N P}^{4}}\left|w_{i j} \varepsilon\right|^{2},
$$

where $w_{i j}$ are numbers of order 1 . As a consequence, the branching ratios of the three transitions $\mu \rightarrow e \gamma, \tau \rightarrow \mu \gamma$, and $\tau \rightarrow e \gamma$ are all expected to be of the same order,

$$
B(\mu \rightarrow e \gamma) \approx B(\tau \rightarrow \mu \gamma) \approx B(\tau \rightarrow e \gamma) .
$$

This is a distinctive feature of this class of models. Given the present experimental bound on $B(\mu \rightarrow e \gamma)$, Eq. (91) implies that $\tau \rightarrow \mu \gamma$ and $\tau \rightarrow e \gamma$ have rates much below the present and expected future sensitivity. Moreover, from the current (future) experimental limit on $B(\mu$ $\rightarrow e \gamma$ ) (Brooks et al., 1999; MEG, 1999; Adam et al., 2010) and assuming $\left|w_{\mu e}\right|=1$, we derive the following bound on $\left|\varepsilon / \Lambda_{N P}^{2}\right|$ :

$$
\begin{aligned}
& B(\mu \rightarrow e \gamma)<1.2 \times 10^{-11}\left(10^{-13}\right), \\
& \left|\frac{\varepsilon}{\Lambda_{N P}^{2}}\right|<1.2 \times 10^{-11}\left(1.1 \times 10^{-12}\right) \mathrm{GeV}^{-2} .
\end{aligned}
$$

Taking two extreme values for the parameter $|\varepsilon|$ we find

$$
\begin{aligned}
& \Lambda_{N P}>20(67) \mathrm{TeV} \quad[|\varepsilon|=0.005], \\
& \Lambda_{N P}>65(210) \mathrm{TeV} \quad[|\varepsilon|=0.05] .
\end{aligned}
$$

This model also allows for four-fermion operators that are not suppressed by any power of the small parameter $t$ or $\epsilon$ and that violate the individual lepton numbers $L_{i}$ (Feruglio and Paris, 2010). They are all characterized by the selection rule $\Delta L_{e} \Delta L_{\mu} \Delta L_{\tau}=2$. For instance, one such operator is

$$
(\bar{l} l)^{\prime}(\bar{l} l)^{\prime \prime}=\left[\bar{l}_{e} l_{\tau} \bar{l}_{\mu} l_{\tau}+\bar{l}_{\mu} l_{e} \bar{l}_{\tau} l_{e}+\bar{l}_{\tau} l_{\mu} \bar{l}_{e} l_{\mu}+\text { H.c. }\right]+\cdots,
$$

where dots stand for additional flavor conserving contributions. These operators can contribute to LFV decays such as $\tau^{-} \rightarrow \mu^{+} e^{-} e^{-}, \tau^{-} \rightarrow e^{+} \mu^{-} \mu^{-}$, and their conjugate, whose branching ratios have upper bounds of the order of $10^{-7}$ (Amsler et al., 2008). Through a rough dimensional estimate we find a lower bound on the scale $\Lambda_{N P}$ of the order of $15 \mathrm{TeV}$. From the previous considerations we see that, even invoking a cancellation in the imaginary part of $\mathcal{Z}_{e e}$ to suppress the contribution to the electron EDM, it is difficult to avoid the conclusion that the scale $\Lambda_{N P}$ should lie considerably above the $\mathrm{TeV}$ range. We recall that if the operator in Eq. (81) originates from one-loop diagrams via the exchange of weakly interacting particles of masses $m_{N P}$, then in our normalization a lower bound on $\Lambda_{N P}$ of $20 \mathrm{TeV}$ corresponds to a lower bound on $m_{N P}$ of about $g_{N P} \Lambda_{N P} /(4 \pi) \approx 1 \mathrm{TeV}$, assuming $g_{N P}$ is similar to the $\mathrm{SU}(2)$ gauge coupling.

All the previous estimates are based on an effective Lagrangian approach, with no explicit reference to the dynamics at the scale $\Lambda_{N P}$. If the degrees of freedom associated to the new physics at the scale $\Lambda_{N P}$ and their interactions are known, it is possible to directly compute the amplitudes of interest. For instance, the SUSY model of Sec. IV can be completed by adding a set of soft SUSY breaking terms, which are constrained by the invariance under $G_{f}=A_{4} \times Z_{3} \times \mathrm{U}(1)_{\mathrm{FN}}$ and its pattern of symmetry breaking (Ishimori et al., 2008; Feruglio et al., 2009a; Hayakawa et al., 2009; Ding and Liu, 2010). LFV amplitudes arise at one-loop level via exchange of sleptons, charginos, and neutralinos with masses of order $m_{\text {SUSY }}$. An explicit computation of $B\left(l_{i} \rightarrow l_{j} \gamma\right)$ confirms both the predictions of Eq. (91) and the behavior of Eq. (90), with $\Lambda_{N P}=(4 \pi / g) m_{\text {SUSY }}$. The coefficients $w_{i j}$ are typically of $\mathcal{O}(0.1)$. When $\varepsilon$ is small, which also entails small tan $\beta$ in our model, relatively light SUSY particles are allowed, while for $\varepsilon$ close to its upper limit, 0.05 , SUSY particle masses of several hundred GeV or close to the $\mathrm{TeV}$ are needed to satisfy the present bound on $B(\mu \rightarrow e \gamma)$ particularly if $\tan \beta$ is larger than 10 . In either case there is only a limited region of the parameter space where it is possible to explain the observed discrepancy in the muon MDM and to satisfy at the same time the current limit on $B(\mu \rightarrow e \gamma)$. An interesting case is that of universal SUSY breaking terms, giving rise to a cancellation in the elements of $\mathcal{Z}_{i j}$ below the diagonal (Feruglio et al., 2009a, 2010). Under these circumstances $B\left(l_{i} \rightarrow l_{j} \gamma\right)$ scale as $\varepsilon^{4}$ rather than as $\varepsilon^{2}$, with the possibility of much lighter SUSY particles. In SUSY $A_{4}$ models also LFV four-fermion operators are depleted by powers of $\varepsilon$ and the corresponding bounds on $m_{\mathrm{SUSY}}$ are relaxed.

In the model discussed in Sec. VIII, with $G_{f}=S_{4} \times Z_{4}$ $\times \mathrm{U}(1)_{\mathrm{FN}}$, the matrix $\mathcal{Z}$ is given by (Masiero, 2009)

$$
\mathcal{Z}=\left(\begin{array}{ccc}
\mathcal{O}\left(t^{2} v^{2}\right) & \mathcal{O}\left(t^{2} v^{2} v^{\prime}\right) & \mathcal{O}\left(t^{2} v^{2} v^{\prime}\right) \\
\mathcal{O}\left(t v v^{\prime}\right) & \mathcal{O}(t v) & \mathcal{O}\left(t v v^{\prime 2}\right) \\
\mathcal{O}\left(v v^{\prime}\right) & \mathcal{O}\left(v v^{\prime 2}\right) & \mathcal{O}(v)
\end{array}\right) .
$$

Predictions for EDMs and MDMs and corresponding bounds are similar to those discussed above in the case of the $A_{4}$ model and summarized in Table VII. Concerning the radiative decays of the charged leptons we find that $R_{\mu e}$ and $R_{\tau e}$ scale as $v^{\prime 2} / \Lambda_{N P}^{4}$, whereas $R_{\tau \mu}$ scales as $v^{\prime 4} / \Lambda_{N P}^{4}$. In this case, the symmetry breaking parameter $v^{\prime}$ is considerably larger than the parameter $\varepsilon$ of the $A_{4}$ model and this gives rise to more restrictive bounds on 
the scale of new physics $\Lambda_{N P}$. From $B(\mu \rightarrow e \gamma)<1.2$ $\times 10^{-11}\left(10^{-13}\right)$ we get

$$
\begin{aligned}
& \Lambda_{N P}>90(300) \mathrm{TeV} \quad\left[v^{\prime}=0.1\right], \\
& \Lambda_{N P}>130(430) \mathrm{TeV} \quad\left[v^{\prime}=0.2\right] .
\end{aligned}
$$

The model also predicts

$$
B(\mu \rightarrow e \gamma) \approx B(\tau \rightarrow e \gamma) \gg B(\tau \rightarrow \mu \gamma) .
$$

Summarizing, in models with discrete flavor symmetries LFV processes are generically suppressed by the presence of small symmetry breaking parameters. However, such a suppression is not completely efficient, at least in the explored models, to guarantee a scale of new physics close to the $\mathrm{TeV}$. The best case is the one of the $A_{4}$ model, thanks to the very small expansion parameter $\varepsilon$. In specific SUSY realizations of the $A_{4}$ symmetry the present limits on the branching ratios of LFV processes still allow for a relatively light spectrum of superparticles in a region of masses of interest to LHC.

\section{LEPTOGENESIS}

The violation of $B-L$ implied by the seesaw mechanism suggests an interesting link between neutrino physics and the mechanism that produced the observed baryon asymmetry in the early Universe. If the baryon asymmetry originates well above the electroweak scale, $B-L$ violation represents a necessary condition since any initial $B+L$ asymmetry would be erased in the subsequent evolution of the Universe. According to leptogenesis the asymmetry is determined by the $C P$ violating out-of-equilibrium decays of the heavy $\mathrm{RH}$ neutrinos (Fukugita and Yanagida, 1986). Through $B-L$ nonconservation of neutrino interactions, the asymmetry is first generated in the leptonic number and then partly converted into the observed baryonic one via sphaleron interactions. Depending on whether the relevant decays occur at a sufficiently high temperature or not, we have an unflavored regime, where the leptons in the final state are indistinguishable, or a flavored regime, where the specific interactions of the different leptons in the decay products cannot be neglected (Abada et al., 2006; Nardi et al., 2006). It is also quite remarkable that, at least in its simplest implementation, leptogenesis requires light neutrino masses below the $\mathrm{eV}$ scale (Büchmuller et al., 2003, 2004, 2005; Giudice et al., 2004) in a range which is fully compatible with other experimental constraints. Unfortunately, without any additional assumptions, it is difficult to promote this elegant picture into a testable theory due to the large number of independent parameters of the seesaw model.

Models of lepton masses based on flavor symmetries typically depend on a restricted number of parameters, thus opening the interesting possibility of relating the baryon asymmetry to other low-energy observables. As a general rule, to provide a realistic description of lepton masses and mixing angles the flavor symmetry should always be broken. The breaking is described by a set of small dimensionless quantities $\varepsilon$, which provide efficient expansion parameters. As seen in previous sections, small observable quantities such as charged-lepton mass ratios, $\theta_{13}, \theta_{23}-\pi / 4$, can be expanded in power series of $\varepsilon$, and the predictions are dominated by the lowest (positive) power.

In the context of leptogenesis, given the extreme smallness of the baryon asymmetry (Komatsu et al., 2009)

$$
\eta_{B}^{\mathrm{CMB}}=(6.2 \pm 0.15) \times 10^{-10},
$$

it can be convenient, at least in a certain regime, that the $C P$ asymmetries in the $\mathrm{RH}$ neutrino decays are also suppressed by powers of $\varepsilon$. If the baryon asymmetry is dominated by the decay of a single RH neutrino, we can write $^{5}$

$$
\eta_{B}=d \xi k,
$$

where $d$ describes the combined effect of sphaleron conversion and dilution from photon production, $\xi$ is the relevant $C P$ asymmetry, and $k$ takes into account the wash-out effects. Typically we expect a dilution factor $d$ of order $10^{-2}$ and, barring fine tuning of the parameters, a wash-out factor $k$ in the range of $10^{-3}-10^{-2}$, which favors $\xi$ around $10^{-6}-10^{-5}$. Such $C P$ asymmetry arises from the interference of the tree-level and the one-loop decay amplitudes and depends quadratically on the neutrino Yukawa couplings. In models such as the ones discussed in Secs. IV and VIII, where the RH neutrino masses are large, close to $10^{14} \mathrm{GeV}$ and the corresponding neutrino Yukawa couplings are of $\mathcal{O}(1)$, a rough estimate of the total $C P$ asymmetry would give $\xi$ $=\mathcal{O}(1 /(8 \pi))$, by far too large compared to $10^{-6}-10^{-5}$. It is therefore interesting to analyze under which conditions the $C P$ asymmetries vanish in the limit of exact symmetry, so that the first nonvanishing contribution is given by some power of the symmetry breaking parameters $\varepsilon$. If the $C P$ asymmetry relevant for leptogenesis is suppressed by powers of $\varepsilon$, this opens the interesting possibility of relating the observed baryon asymmetry $\eta_{B}$ to other low-energy observable quantities (Mohapatra and Nasri, 2005; Mohapatra et al., 2005a; Lin, 2009a) such as $\theta_{13}, \theta_{23}-\pi / 4$, and $B\left(l_{i} \rightarrow l_{j} \gamma\right)$.

The total $C P$ asymmetries in the decay of a RH neutrino $\nu_{i}^{c}$ are

$$
\xi_{i}=\frac{\Gamma_{i}-\bar{\Gamma}_{i}}{\Gamma_{i}+\bar{\Gamma}_{i}},
$$

where $\Gamma_{i}\left(\bar{\Gamma}_{i}\right)$ is the decay rate of $\nu_{i}^{c}$ into leptons (antileptons). In the flavored regime the relevant asymmetries $\xi_{i f}$ involve final states with a specific lepton flavor $f$. The flavored regime takes place for $M_{i} \leqslant c 10^{12} \mathrm{GeV}$ where $c=1\left(1+\tan ^{2} \beta\right)$ in the ordinary (SUSY) case. The

\footnotetext{
${ }^{5}$ We denote the $C P$ asymmetries with $\xi$ and we keep $\varepsilon$ to indicate the generic expansion parameter of a spontaneously broken flavor symmetry.
} 
unflavored regime occurs for RH neutrino masses above that threshold. At one loop we have (Covi et al., 1996)

$$
\begin{aligned}
& \xi_{i f}=\frac{1}{8 \pi \hat{\mathcal{Y}}_{i i}} \sum_{j \neq i}\left\{\operatorname{Im}\left[\hat{\mathcal{Y}}_{i j} \hat{Y}_{i f} \hat{Y}_{j f}^{*}\right] f_{i j}+\operatorname{Im}\left[\hat{\mathcal{Y}}_{j i} \hat{Y}_{i f} \hat{Y}_{j f}^{*}\right] g_{i j}\right\} \\
& \xi_{i}=\sum_{f} \xi_{i f}=\frac{1}{8 \pi \hat{\mathcal{Y}}_{i i}} \sum_{j \neq i} \operatorname{Im}\left[\hat{\mathcal{Y}}_{i j}^{2}\right] f_{i j},
\end{aligned}
$$

where $\mathcal{Y}$ is a combination of the neutrino Yukawa couplings $Y=m_{\nu}^{D} / v_{u}$,

$$
\mathcal{Y}_{i j}=\left(Y Y^{\dagger}\right)_{i j},
$$

and the hat in Eqs. (101) and (102) denotes a basis where the mass matrix $M$ of the heavy Majorana neutrinos and that of charged leptons $m_{l}$ are diagonal. The functions $f_{i j}$ and $g_{i j}$ depend on the mass ratios of the RH neutrino masses $M_{i}$. From Eqs. (101) and (102) we see that both $\xi_{i f}$ and $\xi_{i}$ vanish if $\hat{\mathcal{Y}}$ is diagonal. The total asymmetries $\xi_{i}$ vanish also if $\hat{\mathcal{Y}}$ has real nondiagonal entries. A necessary and sufficient condition for a diagonal $\hat{\mathcal{Y}}$ is

$$
\mathcal{Y} M-M \mathcal{Y}^{T}=0,
$$

where the matrices $\mathcal{Y}$ and $M$ are evaluated in any basis.

If the model is invariant under the action of a flavor symmetry group $G_{f}$, we have an interesting sufficient condition for the vanishing of the $C P$ asymmetries. If the heavy RH neutrinos transform in a (threedimensional) irreducible representation of $G_{f}$, then in the limit of exact symmetry, where the symmetry breaking parameters $\varepsilon$ go to zero, all $C P$ asymmetries vanish (Bertuzzo et al., 2009). In this limit it is possible to show that $\mathcal{Y}$ becomes proportional to the unit matrix as a consequence of a completely general group theoretical property. Thus, from Eqs. (101) and (102) we conclude that the asymmetries $\xi_{i}$ and $\xi_{i f}$ vanish. Note that irreducible representations of dimension larger than 1 are only possible if $G_{f}$ is non-Abelian. Beyond the symmetry limit, in general, $\hat{\mathcal{Y}}$ gets corrections and develops complex off-diagonal entries at some order $\varepsilon^{p}$. If the spectrum of RH neutrinos is nondegenerate in the symmetry limit, we expect $\xi_{i}=\mathcal{O}\left(\varepsilon^{2 p}\right)$ and $\xi_{i f}=\mathcal{O}\left(\varepsilon^{p}\right)$. Degeneracy of RH neutrinos can modify this behavior through the dependence on $\varepsilon$ of the functions $f_{i j}$ and $g_{i j}$. This result applies to both models described in Secs. IV and VIII, where the RH neutrinos transform in the threedimensional representations of $A_{4}$ and $S_{4}$, respectively. In the limit of exact flavor symmetry we find in both cases $\mathcal{Y}=|y|^{2} \mathbf{1}$, where $\mathbf{1}$ denotes the identity matrix. This equality holds in any basis, in particular in the mass eigenstate basis of RH neutrinos and we have $\xi_{i}=0$ in the symmetry limit. In both models all $\mathrm{RH}$ neutrino are very heavy, with masses well above $10^{12} \mathrm{GeV}$, and the unflavored regime applies.

In the $A_{4}$ model of Sec. IV, $\hat{\mathcal{Y}}$ acquires complex offdiagonal entries of order $\varepsilon \approx v_{T} / \Lambda$. The $C P$ asymmetries $\xi_{i}$ depend only on three real parameters: two independent real symmetry breaking parameters $\varepsilon_{i}$ and the lightest neutrino mass. In particular, there is only one independent phase which is determined by the lightest neutrino mass up to an overall sign. We have (Jenkins and Manohar, 2008)

$$
\xi_{i} \approx \frac{\varepsilon^{2}}{8 \pi} .
$$

More precisely (Bertuzzo et al., 2009; Hagedorn et al., 2009; Riva, 2010), for normal ordering of the neutrino mass spectrum all asymmetries $\xi_{i}$ are of the same order of magnitude. For inverted ordering the two asymmetries $\xi_{1,2}$ get enhanced compared to the approximate estimate of Eq. (105) by a factor of $10^{3}$ coming from the functions $f_{12}$ and $f_{21}$ as a result of the near degeneracy of two heavy RH neutrinos. To reproduce the observed baryon asymmetry [Eq. (98)], different wash-out effects are required in the two cases. In the case of normal ordering the experimental value in Eq. (98) is obtained when the parameter $\varepsilon$ is in its natural window, 5 $\times 10^{-3}-5 \times 10^{-2}$, for a wide range of neutrino Yukawa couplings $y$. For inverted ordering a much larger washout suppression is needed. When $\varepsilon$ falls in the optimal range of $5 \times 10^{-3}-5 \times 10^{-2}$ this can be accommodated by restricting both $y \sin \beta$ and $m_{3}$ in a limited range. It is quite remarkable that in both cases the range of the symmetry breaking parameter $\varepsilon$ suggested by the constraints on lepton masses and mixing angles corresponds to that required to get the observed baryon asymmetry through leptogenesis.

In the $S_{4}$ model discussed in Sec. VIII, $\hat{\mathcal{Y}}$ acquires complex off-diagonal entries at the order $v^{4} / v^{\prime}$ and the $C P$ asymmetries are expected to be of order $v^{8} / v^{\prime 2} 8 \pi$. Assuming a typical wash-out suppression of order $10^{-2}$, the observed baryon asymmetry can be obtained for values of $\left(v, v^{\prime}\right)$ close to the range selected to fit chargedlepton masses and mixing angles.

Another class of models where the $C P$ asymmetries vanish is the one of type I seesaw models where the Dirac and Majorana neutrino mass matrix $m_{\nu}^{D}$ and $M$ as well as their seesaw combination $m_{\nu}$ are form diagonalizable. A matrix $A$ depending on a set of parameters $\alpha_{i}$ is said to be form diagonalizable (Low and Volkas, 2003) if it is diagonalized by unitary transformations that do not depend on $\alpha_{i}$,

$$
U_{L}^{\dagger} A(\alpha) U_{R}=A^{d}(\alpha),
$$

where $A^{d}(\alpha)$ is diagonal and the unitary matrices $U_{L, R}$ are independent from $\alpha$. Examples of a formdiagonalizable matrix are $m_{\nu}$ in Eqs. (13), (14), (18), and (19). The parameters are the eigenvalues $m_{1,2,3}$ and the diagonalizing matrices are $U_{R}=U_{L}^{*}=U_{\mathrm{TB}}$ and $U_{R}=U_{L}^{*}$ $=U_{\mathrm{BM}}$, respectively. As we have seen in Sec. II, formdiagonalizable matrices naturally arise in the context of models with discrete flavor symmetries. It is possible to show that if in a type I seesaw $m_{\nu}^{D}, M$, and $m_{\nu}$ are all 
form diagonalizable, then the matrix $\hat{\mathcal{Y}}$ is diagonal and the $C P$ asymmetries vanish (Aristizabal Sierra et al., 2010; González Felipe and Serodio, 2010). In realistic models $m_{\nu}^{D}, M$, and $m_{\nu}$ are typically form diagonalizable only in some symmetry limit. Symmetry breaking terms usually spoil this property and allow for small nonvanishing $C P$ asymmetries.

We have so far discussed the regime of large $\mathrm{RH}$ masses and large neutrino Yukawa couplings. When the smallest RH neutrino mass is below the so-called Davidson-Ibarra bound (Davidson and Ibarra, 2002), 4 $\times 10^{8}-2 \times 10^{9} \mathrm{GeV}$, and we are in the regime of strong hierarchy among $\mathrm{RH}$ neutrino masses, the $C P$ asymmetry associated to the lightest RH neutrino decay is too small to allow for a successful leptogenesis. To evade the Davidson-Ibarra bound we should depart from the strong hierarchical regime. Under certain conditions a significant enhancement of the $C P$ asymmetry can be achieved even for RH neutrino mass ratios as small as 0.1 (Hambye et al., 2004; Raidal et al., 2005). Alternatively, we can exploit the regime of resonant leptogenesis (Pilaftsis, 1997), occurring when the decaying RH neutrino is quasidegenerate in mass with some other RH neutrino, the mass differences being comparable with the RH neutrino decay width. A quasidegeneracy of the $\mathrm{RH}$ neutrino spectrum is better understood and dynamically controlled in the presence of an underlying flavor symmetry. Several symmetries have been proposed in the literature such as $G_{f}=\mathrm{SU}(3)_{e^{c}} \times \mathrm{SU}(3)_{l} \times \mathrm{O}(3)_{\nu^{c}}$ in minimal lepton flavor violation (Cirigliano et al., 2005, 2007; Cirigliano and Grinstein, 2006; Davidson and Palorini, 2006; Branco et al., 2007; Grinstein et al., 2007) or $G_{f}=\mathrm{SO}(3)$ from Pilaftsis and Underwood (2005). In these two examples the light neutrino masses and their mixing angles are not explained but just accommodated. An interesting model based on a flavor symmetry group $G_{f}=A_{4} \times Z_{3} \times Z_{4}$ is that of Branco et al. (2009). Like the model discussed in Sec. IV it predicts a lepton mixing close to TB. Due to the presence of an additional discrete factor in the symmetry group, the $\mathrm{RH}$ neutrino spectrum is degenerate at $\mathrm{LO}$, and the degeneracy is lifted by radiative corrections or small soft breaking terms, allowing for successful resonant leptogenesis, for a wide range of $\mathrm{RH}$ neutrino masses.

\section{SUMMARY AND CONCLUSION}

We reviewed the motivation, formalism, and implications of applying non-Abelian discrete flavor groups to the theory of neutrino mixing. The data on neutrino mixing are by now quite precise. It is a fact that, to a precision comparable with the measurement accuracy, the TB mixing pattern is well approximated by the data (see Fig. 2). If this experimental result is not a mere accident but a real indication that a dynamical mechanism is at work to guarantee the validity of TB mixing in the leading approximation, corrected by small nonleading terms, then non-Abelian discrete flavor groups emerge as the main road to an understanding of this mixing pattern. Indeed, the entries of the TB mixing matrix are clearly suggestive of "rotations" by simple specific angles. In fact, the group $A_{4}$, the simplest group used to explain TB mixing, is specified by the set of those rotations that leave a regular tetrahedron invariant. We started by recalling some basic notions about finite groups and then concentrated on those symmetries, such as $A_{4}$ and $S_{4}$, which are found to be the main candidates for obtaining TB mixing. We discussed the general mechanism that realizes TB mixing within the framework of discrete flavor symmetries. The symmetry is broken down to two different subgroups in the charged-lepton sector and the neutrino sector, and the mixing matrix arises from the mismatch between the two different residual symmetries. TB mixing requires a flavor symmetry group possessing appropriate residual subgroups. The breaking can be realized in a natural way through the specific vacuum alignments of a set of scalar flavons. We described a set of models where TB mixing is indeed derived at leading order within this mechanism. There are many variants of such models (in particular, with or without seesaw) with different detailed predictions for the spectrum of neutrino masses and for deviations from the TB values of the mixing angles. In general at NLO the different mixing angles receive corrections of the same order of magnitude, which are constrained to be small due to the experimental results which are close to the TB values. Indeed, the small experimental error on $\theta_{12}$, which agrees nicely with the value predicted by $\mathrm{TB}$ mixing, suggests that the NLO corrections should be of order of few percent, at most. Additional symmetries are needed, typically of the $\mathrm{U}(1)_{\mathrm{FN}}$ or $Z_{N}$ type, in order to reproduce the mass hierarchy of charged leptons. In the neutrino sector there is no reason for the mass eigenvalues not to be of the same order in absolute value. Thus, the smallness of the ratio $\sqrt{r} \sim 0.2$, where $r$ is defined in Eq. (1), is accidental in most of these models. Both normal and inverse hierarchy spectra can be realized. The phenomenology of the models was summarized. We also discussed the implications of models based on discrete flavor groups for lepton flavor violation and for leptogenesis. Lepton flavor violating processes, the muon $g-2$ and the EDMs of leptons, impose strong constraints on every new physics model. This is also true for the models considered here. However, the specific suppression factors and selection rules induced by the finite flavor symmetry group, in particular by $A_{4}$, may help to improve the consistency of the model even in the presence of new physics at the $\mathrm{TeV}$ scale. The observed baryon asymmetry in the Universe, explained in terms of leptogenesis from the decay of heavy Majorana neutrinos, is found to be compatible with models based on discrete groups. Neutrino Yukawa couplings of order 1 and RH neutrino masses of order $10^{14}-10^{15} \mathrm{GeV}$ would typically lead to $C P$ asymmetries too large to reproduce the observed baryon asymmetry. As a consequence of a general group theoretical property, in all models where the three RH neutrinos transform in a single irreducible representation of the flavor 
group, the unflavored $C P$ asymmetries vanish in the limit of exact symmetry and small values can be generated through NLO corrections.

An obvious question is whether some additional indication for discrete flavor groups can be obtained by considering the extension of the models to the quark sector, perhaps in a grand unified context. The answer appears to be that, while the quark masses and mixings can indeed be reproduced in models where TB mixing is realized in the leptonic sector through the action of discrete groups, there are no specific additional hints in favor of discrete groups that come from the quark sector. Examples of grand unified descriptions of all fermion masses and mixings with TB mixing for neutrinos have been produced and have been discussed in this review. For quarks, only the third generation masses are present at leading order in these models. The other entries of the mass matrices are small due to additional symmetries or other dynamical reasons (for example, suppression factors from extra dimensions), and the small mass ratios and the small mixing angles are generated by these corrective effects and are not due to the discrete group. As a consequence, the action of the discrete flavor group is only manifest among the comparable neutrino sector masses in the basis where charged leptons are diagonal.

Different forms of neutrino mixing other than TB mixing are also amenable to a description in terms of discrete groups. As an alternative to TB mixing, in Sec. VIII we discussed the possibility that actually a more appropriate starting point could be BM mixing, corrected by large terms of $O\left(\lambda_{C}\right)$, with $\lambda_{C}$ being the Cabibbo angle ("weak complementarity"), arising from the diagonalization of charged leptons. By suitably modifying the construction in terms of discrete groups adopted in the case of TB mixing, we identified the group $S_{4}$ as a good candidate to also provide, in a different presentation, the basis for naturally obtaining BM mixing in first approximation. In the model described the NLO terms are such that the dominant corrections only affect $\theta_{12}$ and $\theta_{13}$ [which receive $O\left(\lambda_{C}\right)$ shifts], while $\theta_{23}$ receives smaller corrections. A value of $\theta_{13}$ near the present bound would support this possibility.

In the near future the improved experimental precision on neutrino mixing angles, in particular on $\theta_{13}$, could make the case for TB mixing stronger and then, as a consequence, the case for discrete flavor groups would be also strengthened. Further important input could come from the LHC. In fact, new physics at the weak scale could have important feedback on the physics of neutrino masses and mixing.

\section{ACKNOWLEDGMENTS}

We recognize that this work has been partly supported by the Italian Ministero dell'Università e della Ricerca Scientifica under the COFIN program (PRIN 2008) and by the European Commission under the networks "Heptools" and "Quest for Unification" and Contracts No. MRTN-CT-2006-035505 and No. PITN-GA-2009-237920

\section{REFERENCES}

Abada, A., S. Davidson, F.-X. Josse-Michaux, M. Losada, and A. Riotto, 2006, J. Cosmol. Astropart. Phys. 0604, 004.

Abe, H., K.-S. Choi, T. Kobayashi, and H. Ohki, 2009, Nucl. Phys. B 820, 317.

Abe, K., et al. (Super-Kamiokande), 2006, Phys. Rev. Lett. 97, 171801.

Abe, S., et al. (KamLAND), 2008, Phys. Rev. Lett. 100, 221803. Adam, J., et al. (MEG), 2010, Nucl. Phys. B 834, 1.

Adhikary, B., B. Brahmachari, A. Ghosal, E. Ma, and M. K. Parida, 2006, Phys. Lett. B 638, 345.

Adhikary, B., and A. Ghosal, 2008, Phys. Rev. D 78, 073007.

Adulpravitchai, A., A. Blum, and C. Hagedorn, 2009, J. High

Energy Phys. 03, 046.

Adulpravitchai, A., A. Blum, and M. Lindner, 2009a, J. High

Energy Phys. 07, 053.

Adulpravitchai, A., A. Blum, and M. Lindner, 2009b, J. High

Energy Phys. 09, 018.

Adulpravitchai, A., A. Blum, and W. Rodejohann, 2009, New J. Phys. 11, 063026.

Agafonova, N., et al. (OPERA), 2010, e-print arXiv:1006.1623. Aguilar-Arevalo, A. A., et al. (MiniBooNE), 2009a, Phys. Rev. Lett. 103, 111801.

Aguilar-Arevalo, A. A., et al. (MiniBooNE), 2009b, Phys. Rev. Lett. 102, 101802.

Aharmim, B., et al. (SNO), 2010, Phys. Rev. C 81, 055504.

Ahn, M. H., et al. (K2K), 2006, Phys. Rev. D 74, 072003.

Ahn, Y. H., S. K. Kang, C. S. Kim, and J. Lee, 2006, Phys. Rev. D 73, 093005.

Albright, C. H., and W. Rodejohann, 2008, Phys. Lett. B 665, 378.

Altarelli, G., 2009, Nuovo Cimento 032C, 91.

Altarelli, G., and F. Feruglio, 2004, New J. Phys. 6, 106.

Altarelli, G., and F. Feruglio, 2005, Nucl. Phys. B 720, 64.

Altarelli, G., and F. Feruglio, 2006, Nucl. Phys. B 741, 215.

Altarelli, G., F. Feruglio, and C. Hagedorn, 2008, J. High Energy Phys. 03, 052.

Altarelli, G., F. Feruglio, and Y. Lin, 2007, Nucl. Phys. B 775, 31.

Altarelli, G., F. Feruglio, and I. Masina, 2004, Nucl. Phys. B 689, 157.

Altarelli, G., F. Feruglio, and L. Merlo, 2009, J. High Energy Phys. 05, 020.

Altarelli, G., and D. Meloni, 2009, J. Phys. G 36, 085005.

Amsler, C., et al. (Particle Data Group), 2008, Phys. Lett. B 667, 1.

Antusch, S., S. F. King, and R. N. Mohapatra, 2005, Phys. Lett. B 618, 150.

Antusch, S., S. F. King, and M. Spinrath, 2010, e-print arXiv:1005.0708.

Aranda, A., 2007, Phys. Rev. D 76, 111301.

Aranda, A., C. D. Carone, and R. F. Lebed, 2000a, Phys. Rev. D 62, 016009.

Aranda, A., C. D. Carone, and R. F. Lebed, 2000b, Phys. Lett. B 474, 170.

Aristizabal Sierra, D., F. Bazzocchi, I. de Medeiros Varzielas, L. Merlo, and S. Morisi, 2010, Nucl. Phys. B 827, 34.

Armbruster, B., et al. (KARMEN), 2002, Phys. Rev. D 65, 112001.

Athanassopoulos, C., et al. (LSND), 1996, Phys. Rev. Lett. 77, 3082 .

Athanassopoulos, C., et al. (LSND), 1998a, Phys. Rev. Lett. 81, 
1774.

Athanassopoulos, C., et al. (LSND), 1998b, Phys. Rev. C 58, 2489.

Avignone, F. T., III, S. R. Elliott, and J. Engel, 2008, Rev. Mod. Phys. 80, 481.

Babu, K. S., and S. Gabriel, 2010, e-print arXiv:1006.0203.

Babu, K. S., and X.-G. He, 2005, e-print arXiv:hep-ph/0507217.

Babu, K. S., E. Ma, and J. W. F. Valle, 2003, Phys. Lett. B 552, 207.

Bandyopadhyay, A., S. Choubey, S. Goswami, S. T. Petcov, and D. P. Roy, 2008, e-print arXiv:0804.4857.

Barbieri, R., G. R. Dvali, and L. J. Hall, 1996, Phys. Lett. B 377, 76.

Barbieri, R., L. J. Hall, S. Raby, and A. Romanino, 1997, Nucl. Phys. B 493, 3.

Barbieri, R., L. J. Hall, and A. Romanino, 1997, Phys. Lett. B 401, 47.

Barry, J., and W. Rodejohann, 2010, Phys. Rev. D 81, 093002.

Bazzocchi, F., and I. de Medeiros Varzielas, 2009, Phys. Rev. D 79, 093001.

Bazzocchi, F., M. Frigerio, and S. Morisi, 2008, Phys. Rev. D 78, 116018.

Bazzocchi, F., S. Kaneko, and S. Morisi, 2008, J. High Energy Phys. 03, 063.

Bazzocchi, F., L. Merlo, and S. Morisi, 2009a, Nucl. Phys. B 816, 204.

Bazzocchi, F., L. Merlo, and S. Morisi, 2009b, Phys. Rev. D 80, 053003.

Bazzocchi, F., and S. Morisi, 2009, Phys. Rev. D 80, 096005.

Bazzocchi, F., S. Morisi, and M. Picariello, 2008, Phys. Lett. B 659, 628.

Bazzocchi, F., S. Morisi, M. Picariello, and E. Torrente-Lujan, 2009, J. Phys. G 36, 015002.

Bennett, G. W., et al. (Muon g-2), 2004, Phys. Rev. Lett. 92, 161802.

Berger, J., and Y. Grossman, 2010, J. High Energy Phys. 02, 071.

Bertolini, S., T. Schwetz, and M. Malinsky, 2006, Phys. Rev. D 73, 115012.

Bertuzzo, E., P. Di Bari, F. Feruglio, and E. Nardi, 2009, J. High Energy Phys. 11, 036.

Blum, A., C. Hagedorn, and A. Hohenegger, 2008, J. High Energy Phys. 03, 070.

Blum, A., C. Hagedorn, and M. Lindner, 2008, Phys. Rev. D 77, 076004.

Brahmachari, B., S. Choubey, and M. Mitra, 2008, Phys. Rev. D 77, 073008 .

Branco, G. C., A. J. Buras, S. Jager, S. Uhlig, and A. Weiler, 2007, J. High Energy Phys. 09, 004.

Branco, G. C., R. González Felipe, M. N. Rebelo, and H. Serodio, 2009, Phys. Rev. D 79, 093008.

Brooks, M. L., et al. (MEGA), 1999, Phys. Rev. Lett. 83, 1521.

Büchmuller, W., P. Di Bari, and M. Plümacher, 2003, Nucl. Phys. B 665, 445.

Büchmuller, W., P. Di Bari, and M. Plümacher, 2004, New J. Phys. 6, 105.

Büchmuller, W., P. Di Bari, and M. Plümacher, 2005, Ann. Phys. 315, 305.

Buras, A. J., P. Gambino, M. Gorbahn, S. Jager, and L. Silvestrini, 2001, Phys. Lett. B 500, 161

Burrows, T. J., and S. F. King, 2010, Nucl. Phys. B 835, 174.

Cabibbo, N., 1978, Phys. Lett. B 72, 333.

Cai, Y., and H.-B. Yu, 2006, Phys. Rev. D 74, 115005.
Caravaglios, F., and S. Morisi, 2005a, e-print arXiv:hep-ph/ 0510321.

Caravaglios, F., and S. Morisi, 2005b, e-print arXiv:hep-ph/ 0503234.

Carr, P. D., and P. H. Frampton, 2007, e-print arXiv:hep-ph/ 0701034

Chankowski, P. H., and S. Pokorski, 2002, Int. J. Mod. Phys. A 17, 575 .

Chauhan, B. C., M. Picariello, J. Pulido, and E. TorrenteLujan, 2007, Eur. Phys. J. C 50, 573.

Chen, C.-Y., and L. Wolfenstein, 2008, Phys. Rev. D 77, 093009.

Chen, M.-C., and S. F. King, 2009, J. High Energy Phys. 06, 072.

Chen, M.-C., and K. T. Mahanthappa, 2007, Phys. Lett. B 652, 34.

Chen, S.-L., M. Frigerio, and E. Ma, 2004, Phys. Rev. D 70, 073008.

Chen, S.-L., M. Frigerio, and E. Ma, 2005, Nucl. Phys. B 724, 423.

Cheung, K., S. K. Kang, C. S. Kim, and J. Lee, 2005, Phys. Rev. D 72, 036003.

Ciafaloni, P., M. Picariello, E. Torrente-Lujan, and A. Urbano, 2009, Phys. Rev. D 79, 116010.

Cirigliano, V., and B. Grinstein, 2006, Nucl. Phys. B 752, 18.

Cirigliano, V., B. Grinstein, G. Isidori, and M. B. Wise, 2005, Nucl. Phys. B 728, 121.

Cirigliano, V., G. Isidori, and V. Porretti, 2007, Nucl. Phys. B 763, 228.

Ciuchini, M., G. Degrassi, P. Gambino, and G. F. Giudice, 1998, Nucl. Phys. B 534, 3.

Covi, L., E. Roulet, and F. Vissani, 1996, Phys. Lett. B 384, 169. Cravens, J. P., et al. (Super-Kamiokande), 2008, Phys. Rev. D 78, 032002.

Csaki, C., C. Delaunay, C. Grojean, and Y. Grossman, 2008, J. High Energy Phys. 10, 055.

D'Ambrosio, G., G. F. Giudice, G. Isidori, and A. Strumia, 2002, Nucl. Phys. B 645, 155.

Datta, A., L. Everett, and P. Ramond, 2005, Phys. Lett. B 620, 42.

Davidson, S., and A. Ibarra, 2002, Phys. Lett. B 535, 25. Davidson, S., and F. Palorini, 2006, Phys. Lett. B 642, 72 de Gouvêa, A., 2004, Phys. Rev. D 69, 093007.

del Aguila, F., A. Carmona, and J. Santiago, 2010, e-print arXiv:1001.5151.

de Medeiros Varzielas, I., S. F. King, and G. G. Ross, 2007a, Phys. Lett. B 648, 201.

de Medeiros Varzielas, I., S. F. King, and G. G. Ross, 2007b, Phys. Lett. B 644, 153.

de Medeiros Varzielas, I., and G. G. Ross, 2006, Nucl. Phys. B 733, 31.

Dermisek, R., and S. Raby, 2005, Phys. Lett. B 622, 327.

Dighe, A., S. Goswami, and P. Roy, 2006, Phys. Rev. D 73, 071301.

Ding, G.-J., 2008, Phys. Rev. D 78, 036011.

Ding, G.-J., 2010, Nucl. Phys. B 827, 82.

Ding, G.-J., and J.-F. Liu, 2010, J. High Energy Phys. 05, 029.

Dutta, B., Y. Mimura, and R. N. Mohapatra, 2009, Phys. Rev.

D 80, 095021.

Dutta, B., Y. Mimura, and R. N. Mohapatra, 2010, J. High Energy Phys. 05, 034.

Everett, L. L., and A. J. Stuart, 2009, Phys. Rev. D 79, 085005. Faraggi, A. E., 2001, Phys. Lett. B 520, 337. 
Ferrandis, J., and S. Pakvasa, 2005, Phys. Rev. D 71, 033004.

Feruglio, F., C. Hagedorn, Y. Lin, and L. Merlo, 2007, Nucl. Phys. B 775, 120.

Feruglio, F., C. Hagedorn, Y. Lin, and L. Merlo, 2008, e-print arXiv:0808.0812.

Feruglio, F., C. Hagedorn, Y. Lin, and L. Merlo, 2009a, e-print arXiv:0911.3874.

Feruglio, F., C. Hagedorn, Y. Lin, and L. Merlo, 2009b, Nucl. Phys. B 809, 218.

Feruglio, F., C. Hagedorn, and L. Merlo, 2010, J. High Energy Phys. 03, 084.

Feruglio, F., and Y. Lin, 2008, Nucl. Phys. B 800, 77.

Feruglio, F., and A. Paris, 2010, e-print arXiv:1005.5526.

Feruglio, F., A. Strumia, and F. Vissani, 2002, Nucl. Phys. B 637, 345.

Fogli, G. L., E. Lisi, A. Marrone, A. Palazzo, and A. M. Rotunno, 2008a, Phys. Rev. Lett. 101, 141801.

Fogli, G. L., E. Lisi, A. Marrone, A. Palazzo, and A. M. Rotunno, 2008b, e-print arXiv:0809.2936.

Fogli, G. L., et al., 2008, Phys. Rev. D 78, 033010.

Frampton, P. H., and T. W. Kephart, 1995, Int. J. Mod. Phys. A 10, 4689.

Frampton, P. H., and T. W. Kephart, 2007, J. High Energy Phys. 09, 110.

Frampton, P. H., and S. Matsuzaki, 2008, e-print arXiv:0806.4592.

Frampton, P. H., and S. Matsuzaki, 2009, Phys. Lett. B 679, 347.

Frampton, P. H., and R. N. Mohapatra, 2005, J. High Energy Phys. 01, 025.

Froggatt, C. D., and H. B. Nielsen, 1979, Nucl. Phys. B 147, 277.

Fukugita, M., and T. Yanagida, 1986, Phys. Lett. B 174, 45.

Fukuyama, T., and H. Nishiura, 1997, e-print arXiv:hep-ph/ 9702253.

Ge, S.-F., H.-J. He, and F.-R. Yin, 2010, J. Cosmol. Astropart. Phys. 1005, 017.

Gell-Mann, M., P. Ramond, and R. Slansky, 1979, in Supergravity, edited by P. van Nieuwenhuizen and D. Z. Freedman (North-Holland, Amsterdam, in press).

Ghosal, A., 2003, e-print arXiv:hep-ph/0304090.

Giudice, G. F., A. Notari, M. Raidal, A. Riotto, and A. Strumia, 2004, Nucl. Phys. B 685, 89.

Glashow, S. L., 1980, in Quarks and Leptons, Cargese, edited by M. Levy et al. (Plenum, New York), p. 707.

Goh, H. S., R. N. Mohapatra, and S. Nasri, 2004, Phys. Rev. D 70, 075022.

González Felipe, R., and H. Serodio, 2010, Phys. Rev. D 81, 053008.

Gonzalez-Garcia, M. C., and M. Maltoni, 2008a, Phys. Rep. 460, 1.

Gonzalez-Garcia, M. C., and M. Maltoni, 2008b, Phys. Lett. B 663, 405.

Gonzalez-Garcia, M. C., M. Maltoni, and J. Salvado, 2010, J. High Energy Phys. 04, 056.

Grimus, W., 2006, PoS P2GC, 001.

Grimus, W., A. S. Joshipura, S. Kaneko, L. Lavoura, and M. Tanimoto, 2004, J. High Energy Phys. 07, 078.

Grimus, W., and H. Kuhbock, 2008, Phys. Rev. D 77, 055008. Grimus, W., and L. Lavoura, 2004, J. Phys. G 30, 73.

Grimus, W., and L. Lavoura, 2006, J. High Energy Phys. 01, 018.

Grimus, W., and L. Lavoura, 2008, J. High Energy Phys. 09,
106.

Grimus, W., and L. Lavoura, 2009, J. High Energy Phys. 04, 013.

Grimus, W., L. Lavoura, and P. O. Ludl, 2009, J. Phys. G 36, 115007.

Grimus, W., et al., 2005, Nucl. Phys. B 713, 151.

Grinstein, B., V. Cirigliano, G. Isidori, and M. B. Wise, 2007, Nucl. Phys. B 763, 35.

Haba, N., and K. Yoshioka, 2006, Nucl. Phys. B 739, 254.

Hagedorn, C., S. F. King, and C. Luhn, 2010, e-print arXiv:1003.4249.

Hagedorn, C., M. Lindner, and R. N. Mohapatra, 2006, J. High Energy Phys. 06, 042.

Hagedorn, C., E. Molinaro, and S. T. Petcov, 2009, J. High Energy Phys. 09, 115.

Hall, L. J., and L. Randall, 1990, Phys. Rev. Lett. 65, 2939.

Hambye, T., Y. Lin, A. Notari, M. Papucci, and A. Strumia, 2004, Nucl. Phys. B 695, 169.

Harrison, P. F., D. H. Perkins, and W. G. Scott, 2002, Phys. Lett. B 530, 167.

Harrison, P. F., and W. G. Scott, 2002, Phys. Lett. B 535, 163.

Harrison, P. F., and W. G. Scott, 2003, Phys. Lett. B 557, 76.

Harrison, P. F., and W. G. Scott, 2004, e-print arXiv:hep-ph/ 0402006.

Hayakawa, A., H. Ishimori, Y. Shimizu, and M. Tanimoto, 2009, Phys. Lett. B 680, 334.

He, X.-G., Y.-Y. Keum, and R. R. Volkas, 2006, J. High Energy Phys. 04, 039.

Hirsch, M., A. S. Joshipura, S. Kaneko, and J. W. F. Valle, 2007, Phys. Rev. Lett. 99, 151802.

Hirsch, M., S. Morisi, and J. W. F. Valle, 2008, Phys. Rev. D 78, 093007.

Hirsch, M., J. C. Romao, S. Skadhauge, J. W. F. Valle, and A. Villanova del Moral, 2004, Phys. Rev. D 69, 093006.

Hirsch, M., A. Villanova del Moral, J. W. F. Valle, and E. Ma, 2005, Phys. Rev. D 72, 091301.

Hochmuth, K. A., and W. Rodejohann, 2007, Phys. Rev. D 75, 073001.

Honda, M., and M. Tanimoto, 2008, Prog. Theor. Phys. 119, 583.

Hosaka, J., et al. (Super-Kamiokande), 2006a, Phys. Rev. D 73, 112001.

Hosaka, J., et al. (Super-Kamiokande), 2006b, Phys. Rev. D 74, 032002.

Ishimori, H., T. Kobayashi, Y. Omura, and M. Tanimoto, 2008, J. High Energy Phys. 12, 082.

Ishimori, H., K. Saga, Y. Shimizu, and M. Tanimoto, 2010, e-print arXiv:1004.5004.

Ishimori, H., Y. Shimizu, and M. Tanimoto, 2009, Prog. Theor. Phys. 121, 769.

Ishimori, H., et al., 2010, e-print arXiv:1003.3552.

Jenkins, E. E., and A. V. Manohar, 2008, Phys. Lett. B 668, 210.

Kadosh, A., and E. Pallante, 2010, e-print arXiv:1004.0321.

Kafka, T. (MINOS), 2010, Prog. Part. Nucl. Phys. 64, 184.

Kajiyama, Y., M. Raidal, and A. Strumia, 2007, Phys. Rev. D 76, 117301.

Kaneko, S., H. Sawanaka, T. Shingai, M. Tanimoto, and K. Yoshioka, 2007, Prog. Theor. Phys. 117, 161.

Kang, S. K., C. S. Kim, and J. Lee, 2005, Phys. Lett. B 619, 129.

Kawamura, Y., 2001, Prog. Theor. Phys. 105, 999.

King, S. F., 2005, J. High Energy Phys. 08, 105.

King, S. F., 2010, AIP Conf. Proc. 1200, 103. 
King, S. F., and C. Luhn, 2009a, Nucl. Phys. B 820, 269.

King, S. F., and C. Luhn, 2009b, e-print arXiv:0912.1344.

King, S. F., and M. Malinsky, 2006, J. High Energy Phys. 11, 071.

King, S. F., and M. Malinsky, 2007, Phys. Lett. B 645, 351.

Kitabayashi, T., and M. Yasue, 2003, Phys. Rev. D 67, 015006.

Kitabayashi, T., and M. Yasue, 2005, Phys. Lett. B 621, 133.

Kobayashi, T., H. P. Nilles, F. Ploger, S. Raby, and M. Ratz, 2007, Nucl. Phys. B 768, 135.

Koide, Y., 2004, Phys. Rev. D 69, 093001.

Koide, Y., 2006a, Phys. Rev. D 73, 057901.

Koide, Y., 2006b, Eur. Phys. J. C 48, 223.

Koide, Y., 2007a, e-print arXiv:0707.0899.

Koide, Y., 2007b, Eur. Phys. J. C 50, 809.

Komatsu, E., et al. (WMAP), 2009, Astrophys. J., Suppl. Ser. 180, 330.

Kraus, C., et al., 2005, Eur. Phys. J. C 40, 447.

Kubo, J., 2004, Phys. Lett. B 578, 156.

Kubo, J., A. Mondragon, M. Mondragon, and E. Rodriguez-

Jauregui, 2003, Prog. Theor. Phys. 109, 795.

Lam, C. S., 2001, Phys. Lett. B 507, 214.

Lam, C. S., 2008, Phys. Rev. Lett. 101, 121602.

Lavoura, L., and H. Kuhbock, 2007, Mod. Phys. Lett. A 22, 181.

Lavoura, L., and E. Ma, 2005, Mod. Phys. Lett. A 20, 1217.

Lesgourgues, J., and S. Pastor, 2006, Phys. Rep. 429, 307.

Li, N., and B.-Q. Ma, 2005, Phys. Rev. D 71, 097301.

Lin, Y., 2009a, Phys. Rev. D 80, 076011.

Lin, Y., 2009b, Nucl. Phys. B 813, 91.

Lin, Y., L. Merlo, and A. Paris, 2010, Nucl. Phys. B 835, 238.

Lindner, M., M. A. Schmidt, and A. Y. Smirnov, 2005, J. High Energy Phys. 07, 048.

Low, C. I., and R. R. Volkas, 2003, Phys. Rev. D 68, 033007.

Luhn, C., S. Nasri, and P. Ramond, 2007a, J. Math. Phys. 48, 123519.

Luhn, C., S. Nasri, and P. Ramond, 2007b, J. Math. Phys. 48, 073501.

Luhn, C., S. Nasri, and P. Ramond, 2007c, Phys. Lett. B 652, 27.

Luo, S., and Z.-z. Xing, 2006, Phys. Lett. B 632, 341.

Ma, E., 2004a, Phys. Rev. D 70, 031901.

Ma, E., 2004b, New J. Phys. 6, 104.

Ma, E., 2005a, Phys. Rev. D 72, 037301.

Ma, E., 2005b, Mod. Phys. Lett. A 20, 2767.

Ma, E., 2005c, Mod. Phys. Lett. A 20, 2601.

Ma, E., 2006a, Phys. Lett. B 632, 352.

Ma, E., 2006b, Mod. Phys. Lett. A 21, 2931.

Ma, E., 2006c, Phys. Rev. D 73, 057304.

Ma, E., 2007, Mod. Phys. Lett. A 22, 101.

Ma, E., 2008, Phys. Lett. B 660, 505.

Ma, E., and M. Raidal, 2001, Phys. Rev. Lett. 87, 011802.

Ma, E., and G. Rajasekaran, 2001, Phys. Rev. D 64, 113012.

Ma, E., H. Sawanaka, and M. Tanimoto, 2006, Phys. Lett. B 641, 301.

Maltoni, M., and T. Schwetz, 2008, PoS IDM 2008, 072.

Masiero, L., 2009, Graduation thesis (University of Padova).

Matias, J., and C. P. Burgess, 2005, J. High Energy Phys. 09, 052.

MEG, 1999, Proposal of the MEG experiment at http:// meg.web.psi.ch

Meloni, D., 2010, J. Phys. G 37, 055201.

Minakata, H., 2005, e-print arXiv:hep-ph/0505262.

Minakata, H., and A. Y. Smirnov, 2004, Phys. Rev. D 70,
073009.

Minkowski, P., 1977, Phys. Lett. B 67, 421.

Mohapatra, R. N., and S. Nasri, 2005, Phys. Rev. D 71, 033001.

Mohapatra, R. N., S. Nasri, and H.-B. Yu, 2005a, Phys. Lett. B 615, 231.

Mohapatra, R. N., S. Nasri, and H.-B. Yu, 2005b, Phys. Rev. D 72, 033007.

Mohapatra, R. N., S. Nasri, and H.-B. Yu, 2006a, Phys. Lett. B 636, 114.

Mohapatra, R. N., S. Nasri, and H.-B. Yu, 2006b, Phys. Lett. B 639, 318.

Mohapatra, R. N., and S. Nussinov, 1999, Phys. Rev. D 60, 013002.

Mohapatra, R. N., N. Okada, and H.-B. Yu, 2007, Phys. Rev. D 76, 015013.

Mohapatra, R. N., M. K. Parida, and G. Rajasekaran, 2004, Phys. Rev. D 69, 053007.

Mohapatra, R. N., and W. Rodejohann, 2005, Phys. Rev. D 72, 053001.

Mohapatra, R. N., and G. Senjanovic, 1980, Phys. Rev. Lett. 44, 912.

Mohapatra, R. N., and A. Y. Smirnov, 2006, Annu. Rev. Nucl. Part. Sci. 56, 569.

Mohapatra, R. N., et al., 2007, Rep. Prog. Phys. 70, 1757.

Morisi, S., 2006, e-print arXiv:hep-ph/0604106.

Morisi, S., 2009, Phys. Rev. D 79, 033008.

Morisi, S., and E. Peinado, 2010, Phys. Rev. D 81, 085015.

Morisi, S., and M. Picariello, 2006, Int. J. Theor. Phys. 45, 1267.

Morisi, S., M. Picariello, and E. Torrente-Lujan, 2007, Phys.

Rev. D 75, 075015.

Nardi, E., Y. Nir, E. Roulet, and J. Racker, 2006, J. High Energy Phys. 01, 164.

Ohlsson, T., 2005, Phys. Lett. B 622, 159.

Passera, M., W. J. Marciano, and A. Sirlin, 2009, AIP Conf. Proc. 1078, 378.

Picariello, M., 2008, Int. J. Mod. Phys. A 23, 4435.

Pilaftsis, A., 1997, Phys. Rev. D 56, 5431.

Pilaftsis, A., and T. E. J. Underwood, 2005, Phys. Rev. D 72, 113001.

Plentinger, F., and G. Seidl, 2008, Phys. Rev. D 78, 045004.

Plentinger, F., G. Seidl, and W. Winter, 2007, Phys. Rev. D 76, 113003.

Plentinger, F., G. Seidl, and W. Winter, 2008, Nucl. Phys. B 791, 60.

Pomarol, A., and D. Tommasini, 1996, Nucl. Phys. B 466, 3.

Raidal, M., 2004, Phys. Rev. Lett. 93, 161801.

Raidal, M., A. Strumia, and K. Turzynski, 2005, Phys. Lett. B 609, 351.

Raidal, M., et al., 2008, Eur. Phys. J. C 57, 13.

Riva, F., 2010, e-print arXiv:1004.1177.

Rodejohann, W., 2009, Phys. Lett. B 671, 267.

Schmidt, M. A., and A. Y. Smirnov, 2006, Phys. Rev. D 74, 113003.

Schwetz, T., M. A. Tortola, and J. W. F. Valle, 2008, New J. Phys. 10, 113011.

Sekhar Chivukula, R., and H. Georgi, 1987, Phys. Lett. B 188, 99.

Strumia, A., and F. Vissani, 2006, e-print arXiv:hep-ph/ 0606054.

Tanimoto, M., and T. Yanagida, 2006, Phys. Lett. B 633, 567.

Teshima, T., 2006, Phys. Rev. D 73, 045019.

Toorop, R. d. A., F. Bazzocchi, and L. Merlo, 2010, e-print arXiv:1003.4502. 
Weinberg, S., 1979, Phys. Rev. Lett. 43, 1566.

Witten, E., 1985, Nucl. Phys. B 258, 75.

Wolfenstein, L., 1978, Phys. Rev. D 18, 958.

Xing, Z.-Z., 2002, Phys. Lett. B 533, 85.

Xing, Z.-z., 2005, Phys. Lett. B 618, 141.

Yanagida, T., 1979, Horizontal Symmetry and Masses of Neu- trinos, Proceedings of the Workshop on the Baryon Number of the Universe and Unified Theories (Tsukuba Workshop, Tsukuba).

Yin, F., 2007, Phys. Rev. D 75, 073010.

Zee, A., 2005, Phys. Lett. B 630, 58.

Zhang, H., 2007, Phys. Lett. B 655, 132. 